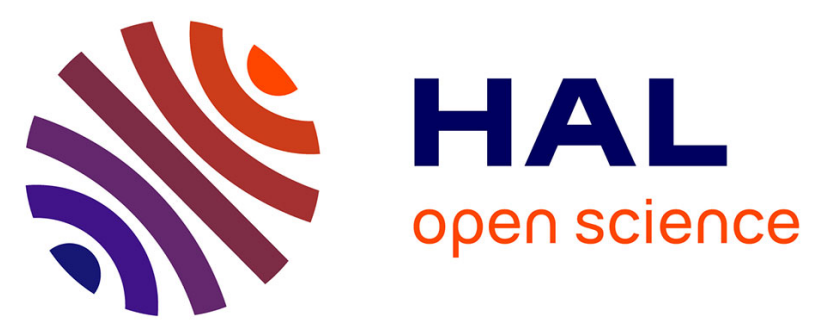

\title{
AN ENTROPIC INTERPOLATION PROBLEM FOR INCOMPRESSIBLE VISCOUS FLUIDS
}

Marc Arnaudon, Ana Bela Cruzeiro, Christian Léonard, Jean-Claude Zambrini

\section{To cite this version:}

Marc Arnaudon, Ana Bela Cruzeiro, Christian Léonard, Jean-Claude Zambrini. AN ENTROPIC INTERPOLATION PROBLEM FOR INCOMPRESSIBLE VISCOUS FLUIDS. Annales de l'Institut Henri Poincaré, Probabilités et Statistiques, 2020, 56 (3), 10.1214/19-AIHP1036 . hal-02179693

\section{HAL Id: hal-02179693 https://hal.science/hal-02179693}

Submitted on 11 Jul 2019

HAL is a multi-disciplinary open access archive for the deposit and dissemination of scientific research documents, whether they are published or not. The documents may come from teaching and research institutions in France or abroad, or from public or private research centers.
L'archive ouverte pluridisciplinaire HAL, est destinée au dépôt et à la diffusion de documents scientifiques de niveau recherche, publiés ou non, émanant des établissements d'enseignement et de recherche français ou étrangers, des laboratoires publics ou privés. 


\title{
AN ENTROPIC INTERPOLATION PROBLEM FOR INCOMPRESSIBLE VISCOUS FLUIDS
}

\author{
MARC ARNAUDON, ANA BELA CRUZEIRO, CHRISTIAN LÉONARD, \\ AND JEAN-CLAUDE ZAMBRINI
}

\begin{abstract}
In view of studying incompressible inviscid fluids, Brenier introduced in the late 80's a relaxation of a geodesic problem addressed by Arnold in 1966. Instead of inviscid fluids, the present paper is devoted to incompressible viscous fluids. A natural analogue of Brenier's problem is introduced, where generalized flows are no more supported by absolutely continuous paths, but by Brownian sample paths. It turns out that this new variational problem is an entropy minimization problem with marginal constraints entering the class of convex minimization problems.

This paper explores the connection between this variational problem and Brenier's original problem. Its dual problem is derived and the general form of its solution is described. Under the restrictive assumption that the pressure is a nice function, the kinematics of its solution is made explicit and its relation with viscous fluid dynamics is discussed.
\end{abstract}

\section{Contents}

Introduction

1. Notation and outline of the paper 2

2. Brenier-Schrödinger problem $\quad 5$

3. Duality 11

4. General shape of the solution 16

5. Kinematics of regular solutions 18

6. Existence of a solution on $\mathbb{T}^{n} \quad 24$

7. Open problems 26

$\begin{array}{ll}\text { References } & 27\end{array}$

\section{INTRODUCTION}

In the article [Arn66], Arnold addressed a geodesic problem on the manifold of all volume preserving diffeomorphisms on the torus. The resulting geodesics offer us a natural description of the evolution of an incompressible perfect fluid. Unfortunately, very little is known on the global existence of these geodesics [EM70] and Shnirelman proved in [Shn85, Shn94] that solutions do not exist in general. In the seminal article [Bre89b], Brenier introduced a relaxation of Arnold's problem in terms of generalized flows. In this extended setting, global existence of generalized "minimizing flows" is much easier to obtain.

While both Arnold's and Brenier's problems are related to the evolution of inviscid fluids, usually described by the Euler equation (5), the present article introduces a stochastic

Date: July 5th, 2019.

Key words and phrases. Incompressible viscous fluids, entropy minimization, diffusion processes, convex duality, stochastic velocities. 
analogue of Brenier's relaxed problem related to the description of the evolution of viscous fluids. This viscosity is usually modeled by adding an extra Laplacian term to the Euler equation, leading to the Navier-Stokes equation (9). Following Itô's stochastic description of parabolic diffusion equations, we shall introduce stochastic differential equations based on Brownian motion to take the viscosity into account.

Brenier's problem (8) amounts to minimize an average kinetic action, the averaging procedure being performed over the set of all absolutely continuous sample paths. This is no longer available when the relevant sample paths are nowhere differentiable Brownian trajectories. However, it is still possible to consider some stochastic action whose kinetic energy involves Nelson regularized derivatives. It happens that this stochastic action is a relative entropy (with respect to the Wiener measure) and that Brenier's minimization of an average kinetic action turns out to be an entropy minimization problem: the Brenier-Schrödinger (shortly BS) problem (BS) stated below at page 10. After our initial manuscript, this problem was studied by other authors ([BCN17], [B], [BM]) where it is referred as the "Bredinger" and more recently as "Brödinger" problem.

It is a central object of the PhD Theses of L. Nenna [Nen16] and A. Baradat [Ba19].

Literature. Brenier's relaxation of Arnold's geodesic problem was introduced in [Bre89a]. Its dual problem was established and investigated for the first time in [Bre93] where it was emphasized that the pressure field is the natural Lagrange multiplier of the incompressibility condition. The connection between solutions to Brenier's problem and the notion of measure-valued solutions to the Euler equation in the sense of DiPerna and Majda was clarified in [Bre99]. The regularity of the pressure field was explored in [Bre93] and revisited in [Bre99]. Further improvements about the dual problem and the regularity of the pressure were obtained later by Ambrosio and Figalli in [AF08, AF09].

Considering viscous fluids (Navier-Stokes equation) instead of inviscid ones, we refer to [AAC14, ACF] for works where generalized flows are also considered. The present article is also about generalized flows in the setting of viscous fluid but with an alternate point of view. To our knowledge, it is the first attempt to extend Brenier's approach in the context of viscous fluids.

Simulations for our results can be obtained with the method developed in [BCN17] (see Remark 4.9).

\section{Notation And OUtline of the PAPER}

The sets of all Borel probability measures on a topological set $\mathcal{Z}$ is denoted by $\mathrm{P}(\mathcal{Z})$. For any probability measure $m \in \mathrm{P}(A)$ on the Borel set $A$, the push-forward of $m$ by the Borel measurable mapping $\theta: A \rightarrow B$ is denoted by $\theta_{\#} m \in \mathrm{P}(B)$ and defined by $\theta_{\#} m(d b):=m\left(\theta^{-1}(d b)\right)$, for any Borel subset $d b \subset B$.

State space. In general the state space $\mathcal{X}$ for the fluid will be either the flat torus $\mathcal{X}=$ $\mathbb{T}^{n}=\mathbb{R}^{n} / \mathbb{Z}^{n}$ or the whole space $\mathcal{X}=\mathbb{R}^{n}$. We denote it by $\mathcal{X}$ when its specific structure does not matter.

Path space. We denote $\Omega:=C([0,1], \mathcal{X})$ the set of all continuous paths from the unit time interval $[0,1]$ to $\mathcal{X}$. As usual, the canonical process is defined by

$$
X_{t}(\omega)=\omega_{t} \in \mathcal{X}, \quad t \in[0,1], \omega=\left(\omega_{s}\right)_{0 \leq s \leq 1} \in \Omega
$$

and $\Omega$ is equipped with the canonical $\sigma$-field $\sigma\left(X_{t} ; 0 \leq t \leq 1\right)$. 
Marginal measures. For any $Q \in \mathrm{P}(\Omega)$ and $0 \leq s, t \leq 1$, we denote $Q_{t}=\left(X_{t}\right)_{\#} Q \in \mathrm{P}(\mathcal{X})$ and $Q_{s t}=\left(X_{s}, X_{t}\right)_{\#} Q \in \mathrm{P}\left(\mathcal{X}^{2}\right)$; they are respectively the laws of the position $X_{t}$ at time $t$ and of the couple of positions $\left(X_{s}, X_{t}\right)$ when the law of the whole random trajectory is $Q$. In particular, taking $s=0$ and $t=1, Q_{01}:=\left(X_{0}, X_{1}\right)_{\#} Q \in \mathrm{P}\left(\mathcal{X}^{2}\right)$ is the endpoint projection of $Q$ onto $\mathcal{X}^{2}$. If $Q$ describes the random behavior of a particle, then $Q_{t}$ and $Q_{01}$ describe respectively the random behaviors of the particle at time $t$ and the couple of endpoint positions $\left(X_{0}, X_{1}\right)$. We denote $Q^{x}:=Q\left(\cdot \mid X_{0}=x\right) \in \mathrm{P}(\Omega)$ and the bridge of $Q$ between $x$ and $y \in \mathcal{X}$ is $Q^{x y}(\cdot):=Q\left(\cdot \mid X_{0}=x, X_{1}=y\right) \in \mathrm{P}(\Omega)$. In particular, as $\mathcal{X}$ is Polish, the following disintegration formulas

$$
Q(\cdot)=\int_{\mathcal{X}} Q^{x}(\cdot) Q_{0}(d x)=\int_{\mathcal{X}^{2}} Q^{x y}(\cdot) Q_{01}(d x d y) \in \mathrm{P}(\Omega)
$$

are meaningful, i.e. $x \in \mathcal{X} \mapsto Q^{x} \in \mathrm{P}(\Omega)$ and $(x, y) \in \mathcal{X}^{2} \mapsto Q^{x y} \in \mathrm{P}(\Omega)$ are measurable kernels.

Relative entropy. Let $\mathcal{Y}$ be a measurable space. We denote $\mathrm{P}(\mathcal{Y})$ and $\mathrm{M}(\mathcal{Y})$ respectively the sets of probability and positive measures on $\mathcal{Y}$. The relative entropy of $q \in \mathrm{M}(\mathcal{Y})$ with respect to the reference measure $r \in M(\mathcal{Y})$ is defined by

$$
H(\mathrm{q} \mid \mathrm{r}):=\int_{\mathcal{Y}} \log \left(\frac{d \mathbf{q}}{d \mathbf{r}}\right) d \mathbf{q} \in(-\infty, \infty]
$$

whenever the integral is meaningful, i.e. when $q$ is absolutely continuous with respect to $\mathbf{r}$ and $\int_{\mathcal{Y}} \log _{-}(d \mathbf{q} / d \mathbf{r}) d \mathbf{q}<\infty$.

When $r \in \mathrm{P}(\mathcal{Y})$ is a probability measure, for all probability measures $\mathrm{q} \in \mathrm{P}(\mathcal{Y})$, we have

$$
H(\mathrm{q} \mid \mathrm{r})=\min H(\cdot \mid \mathrm{r})=0 \Longleftrightarrow \mathrm{q}=\mathrm{r} .
$$

A frequent use will be made of the additive decomposition formula

$$
H(\mathbf{q} \mid \mathbf{r})=H\left(f_{\#} \mathbf{q} \mid f_{\#} \mathbf{r}\right)+\int_{\mathcal{Z}} H\left(\mathbf{q}^{f=z} \mid \mathbf{r}^{f=z}\right)\left(f_{\#} \mathbf{q}\right)(d z)
$$

where $f: \mathcal{Y} \rightarrow \mathcal{Z}$ is any measurable mapping between the Polish spaces $\mathcal{Y}$ and $\mathcal{Z}$ equipped with their Borel $\sigma$-fields and $\mathbf{q}^{f=z}$ is a regular version of the conditioned probability measure $\mathrm{q}(\cdot \mid f=z)$. For a detailed account on this decomposition, see for instance [GL10, Appendix].

- Note that as a definition $\mathrm{q}^{f=z}$ is always a probability measure, even when $\mathrm{q}$ is not.

- It is necessary that $f_{\#} \mathbf{q}$ is $\sigma$-finite for the conditional probability measure $\mathbf{q}^{f}$ to be defined properly.

In particular, since the relative entropy of two probability measures is nonnegative, we see with (1) and (2) that

$$
H\left(f_{\#} \mathrm{p} \mid f_{\#} \mathrm{r}\right) \leq H(\mathrm{q} \mid \mathrm{r}),
$$

expressing the well-known property of decrease of the entropy by measurable push-forward. Taking $f=X_{0}: \Omega \rightarrow \mathcal{X}$ in (2) gives, for any $Q, R \in \mathrm{M}(\Omega)$,

$$
H(Q \mid R)=H\left(Q_{0} \mid R_{0}\right)+\int_{\mathcal{X}} H\left(Q^{x} \mid R^{x}\right) Q_{0}(d x)
$$


whenever $Q_{0}$ and $R_{0}$ are $\sigma$-finite and $H\left(Q_{0} \mid R_{0}\right)$ makes sense in $(-\infty, \infty]$. An interesting situation where unbounded path measures arise naturally is when the initial marginals

$$
Q_{0}=R_{0}=\mathrm{vol}
$$

are prescribed to be the volume measure on $\mathcal{X}=\mathbb{R}^{n}$. In this case,

$$
H(Q \mid R)=\int_{\mathcal{X}} H\left(Q^{x} \mid R^{x}\right) \operatorname{vol}(d x) .
$$

Outline of the paper. The BS-problem (BS) consists in finding a measure $Q$ on path space $\Omega$ minimizing $H(Q \mid R)$ under the constraint $Q_{t}=\mu_{t}$ for all $t \in \mathcal{T}$ and $Q_{01}=\pi$, where $\mathcal{T}, \mu_{t}$ and $\pi$ are prescribed, $\mathcal{T}$ being a measurable subset of $[0,1], \mu_{t}$ measures on $\mathcal{X}, \pi$ a measure on $\mathcal{X} \times \mathcal{X}$. More about the connection between BS and Brenier problems and their relations with fluid dynamics is given at Section 2. In Proposition 2.3 we prove that the BS minimization problem (BS) admits a solution if and only if there exists some probability measure $Q$ on the path space such that $H(Q \mid R)<\infty$ and satisfying all the constraints in (BS).

Since the BS-problem enters the class of convex minimization problems, it admits a natural dual problem; this is exposed at Section 3. It is the place where the pressure enters the game. In Proposition 3.3 we state the problem dual to the BS-problem (BS), coming from the well-known abstract duality result Theorem 3.4. The pressure naturally appears as a Lagrange multiplier. In Corollary 3.7 we establish a formula for the minimizing probability $P$ using the duality in the case where both the primal and dual problems are attained.

The general form of Radon-Nikodym density of the solution of the BS-problem with respect to the Wiener measure is described at Section 4 and more precisely in Theorem 4.7. It is shown that, in general, this solution fails to be Markov but remains reciprocal, an extension of Markov property due, in substance, to Schrödinger and Bernstein.

At Section 5, under the restrictive assumption that the pressure is a nice function, the kinematics of the solution is made explicit. This permits us to establish a connection between the BS-problem and fluid dynamics, under a of superposition or multi-phase principle (cf. Remark 5.7). We prove in Theorem 5.3 that conditioned to a starting point, the velocity field is the gradient of a potential satisfying a second-order Hamilton-Jacobi equation. The main tool for the proof is the identification of the change of probability measures coming from the duality problem with the one coming from Girsanov formula. The equation obtained with the forward velocity is not Navier-Stokes equation: the diffusive term has the wrong sign. So we do again the same work but using backward velocity, and prove that this velocity conditioned to arrival point solves the Navier-Stokes equation and is the gradient of a potential satisfying some Hamilton-Jacobi-Bellman equation. When the marginals of the probability $P$ are constant and equal to Lebesgue measure, the current velocity (the half sum of unconditioned forward and backward velocity) is divergence-free. The volume constraint is recovered superposing flows tagged by their final positions, which are gradient flows. On the other hand, the unconditioned velocities are not gradient fields. Another remarkable fact is that the pressure in our HamiltonJacobi-Bellman equations is the same for all conditioned flows. This is fully analogous to the properties of the pressure in Brenier's problem (8), see [Bre89b].

Finally, the last Section 6 is devoted to a characterization of the existence of a solution to the BS-problem when the state space is the torus $\mathbb{T}^{n}$ or a compact symmetric space. We prove in Proposition 6.1 that as soon as the constraint on joint initial and final law $\pi$ has finite relative entropy with respect to the reference measure $R_{01}$, then there exists a 
probability measure on the path space with finite relative entropy with respect to $R$. As a consequence, we obtain in Corollary 6.2 (which directly uses Proposition 2.3) that the entropy minimization problem on compact symmetric space admits a unique solution if and only if $H\left(\pi \mid R_{01}\right)<\infty$. For proving Proposition 6.1 , invariance of $R$ by the action of the group of isommetries together with transitivity of this group are the main ingredients, which allow to construct some probability $Q$ with $Q_{01}=\pi$ and $H(Q \mid R)<\infty$, by letting the conditional probabilities $Q^{x y}=\int_{\mathcal{X}} R^{x y}\left(\cdot \mid X_{1 / 2}=z\right) \operatorname{vol}(d z)$.

In the present article, the difficult problem of the regularity of the pressure field is left apart, see the discussion below (30).

\section{BRENIER-SCHRÖDINGER PROBLEM}

The main role of this article is played by the BS-problem, an entropy minimization problem stated below at (BS). The present section is devoted to a brief exposition of some relations between the BS-problem and the evolution of an incompressible viscous fluid. As our approach follows Brenier's one, we start with Brenier's problem and its relation with the evolution of an incompressible inviscid fluid.

Eulerian and Lagrangian coordinates. These coordinates correspond to two different descriptions of the same fluid flow through space and time. Let the state space $\mathcal{X}$ be a subset of $\mathbb{R}^{n}$.

(1) The Eulerian specification of the flow field in $\mathcal{X}$ is a vector field

$$
(t, x) \in[0,1] \times \mathcal{X} \mapsto v(t, x) \in \mathbb{R}^{n}
$$

giving the velocity at position $x$ and time $t$. One looks at the fluid motion focusing on specific locations in $\mathcal{X}$.

(2) The Lagrangian specification of the flow field is a function

$$
(t, x) \in[0,1] \times \mathcal{X} \mapsto q(t, x) \in \mathcal{X}
$$

giving the position at time $t$ of the parcel which was located at $x$ at time $t=0$. One looks at fluid motion following an individual particle. The labeling of the fluid particles allows keeping track of the changes of the shape of fluid parcels over time.

The two specifications are related by: $v(t, q(t, x))=\partial_{t} q(t, x)$. The total rate of change of a function or a vector field $F(t, z)$ experienced by a specific flow parcel is

$$
\mathrm{D}_{t} F(t, z)=\left.\frac{d}{d t} F(t, q(t, x))\right|_{x=q_{t}^{-1}(z)}
$$

where $z$ is fixed. This gives $\mathrm{D}_{t} F=\left(\partial_{t}+v \cdot \nabla\right) F$, since

$$
\begin{aligned}
\frac{d}{d t} F(t, q(t, x))_{x_{x=q_{t}^{-1}(z)}} & =\partial_{t} F(t, q(t, x))_{{ }_{x=q_{t}^{-1}(z)}}+\left.\partial_{t} q(t, x) \cdot \nabla F(t, q(t, x))\right|_{x=q_{t}^{-1}(z)} \\
& =\partial_{t} F(t, z)+v(t, z) \cdot \nabla F(t, z) .
\end{aligned}
$$

This formula is meaningful if for each $t$, the map $x \mapsto q(t, x)$ is injective. The operator

$$
\mathrm{D}_{t}=\partial_{t}+v \cdot \nabla
$$

is sometimes called the convective derivative. 
Euler equation. Let $\mathcal{X}$ be a bounded domain of $\mathbb{R}^{n}$. A fluid in $\mathcal{X}$ is said to be incompressible if the volume is preserved along the flow. This is equivalent to

$$
\nabla \cdot v=0
$$

that is the divergence of the velocity field $v$ vanishes everywhere. If the domain $\mathcal{X}$ has a boundary $\partial \mathcal{X}$, the impermeability condition is

$$
n \cdot v=0
$$

where $n$ is a normal vector to $\partial \mathcal{X}$. From now on, we shall restrict our attention to domains $\mathcal{X}$ without boundary so that the impermeability condition is dropped down.

The Euler equation is Newton's equation of motion

$$
\mathrm{D}_{t} v=-\nabla p
$$

where $\mathrm{D}_{t} v$ is the convective acceleration and the scalar pressure field $p:[0,1] \times \mathcal{X} \rightarrow \mathbb{R}$ is part of the solution to be found out with $v$. The force $-\nabla p$ is necessary for the volume to be preserved as time evolves. The fluid moves from high pressure to low pressure areas. Because of the expression (4) of the convective derivative, we obtain

$$
\begin{cases}\partial_{t} v+v \cdot \nabla v+\nabla p=0, & t \geq 0, x \in \mathcal{X} \\ \nabla \cdot v=0, & t \geq 0, x \in \mathcal{X} \\ v(0, \cdot)=v_{0}, & t=0\end{cases}
$$

which is the Euler equation of the unknown $(v, p)$ for an incompressible fluid seen as a Cauchy problem with a given initial velocity field $v_{0}$.

Arnold's flow of diffeomorphisms. The Cauchy problem (5) is notoriously difficult and there is some hope to understand it a little further by considering a variant which is closer to a variational approach of classical mechanics. Arnold [Arn66] proposed to look at the following fixed endpoint version of the Cauchy problem (5):

$$
\begin{cases}\partial_{t} v+v \cdot \nabla v+\nabla p=0, & 0 \leq t \leq 1 \\ \nabla \cdot v=0, & 0 \leq t \leq 1 \\ q_{1}[v]=h & \end{cases}
$$

where $\mathcal{X}$ is a compact manifold with no boundary, typically $\mathcal{X}=\mathbb{T}^{n}$,

- $q_{1}[v]$ is defined by $q_{1}[v](x):=\omega_{1}^{x}, x \in \mathcal{X}$, with $\left\{\begin{array}{ll}\dot{\omega}_{t}^{x}=v\left(t, \omega_{t}^{x}\right), & 0 \leq t \leq 1 \\ \omega_{0}^{x}=x, & t=0\end{array}\right.$;

- $h$ belongs to the group $G_{\mathrm{vol}}:=\{g$ diffeo: $\operatorname{det} \mathrm{D} g=1\}$ of all volume and orientation preserving diffeomorphisms of $\mathcal{X}$.

This should be regarded informally since it is implicitly assumed in the definition of $q_{1}[v]$ that the field $v$ admits a unique integral curve for each starting point $x$. In fact, the exact purpose of [Arn66] is to describe the fluid evolution by means of pathlines $\left(q_{t}(\cdot)\right)_{0 \leq t \leq 1}$ which are seen as trajectories in $G_{\mathrm{vol}}$. One can prove that any solution $\left(q_{t}(\cdot)\right)_{0 \leq t \leq 1}$ of the action minimizing problem

$$
\int_{[0,1] \times \mathcal{X}}\left|\partial_{t} q_{t}(x)\right|^{2} d x d t \rightarrow \min : \quad q_{t}(\cdot) \in G_{\mathrm{vol}}, \forall 0 \leq t \leq 1, \quad q_{0}(\cdot)=\operatorname{Id}, q_{1}(\cdot)=h
$$

where $h$ is a prescribed element of $G_{\mathrm{vol}}$, is such that the velocity field $v(t, z)=\partial_{t} q_{t}\left(q_{t}^{-1}(z)\right)$ is a solution of (6) for some pressure field $p$. This minimizer is nothing but a geodesic flow on $G_{\text {vol }}$ with prescribed endpoint positions Id and $h$. The pressure $p$ disappears from the picture since $-\nabla p$ can be seen as the force necessary to maintain the motion on 
the manifold $G$ of all diffeomorphisms inside the submanifold $G_{\text {vol }}$ of volume preserving diffeomorphisms (the orientation is automatically preserved by continuity of the motion).

Brenier's generalized flow. Solving the geodesic problem (7) in $G_{\mathrm{vol}}$ remains difficult. Indeed, the only known attempt is done in [EM70] where a solution is proved to exist for $h$ very close to the identity. Actually there are examples where such geodesics do not exist, see [Shn85, Shn94]. Therefore, Brenier [Bre89b] relaxed (7) by introducing a probabilistic representation. Brenier's problem consists of minimizing an average kinetic action subject to incompressibility and endpoint constraints. It is

$$
E_{Q} \int_{[0,1]}\left|\dot{X}_{t}\right|^{2} d t \rightarrow \min ; \quad Q \in \mathrm{P}(\Omega):\left[Q_{t}=\text { vol, } \forall 0 \leq t \leq 1\right], Q_{01}=\pi
$$

where $\pi \in \mathrm{P}\left(\mathcal{X}^{2}\right)$ is a prescribed bistochastic probability measure, i.e. its marginals satisfy $\pi_{0}=\pi_{1}=$ vol and $\dot{X}_{t}(\omega)=\dot{\omega}_{t}$ for any absolutely continuous path $\omega \in \Omega$ with generalized time derivative $\dot{\omega}$. In the above action functional, it is understood that $\int_{[0,1]}\left|\dot{\omega}_{t}\right|^{2} d t=\infty$ whenever $\omega \in \Omega$ is not absolutely continuous. Therefore any solution $P$ of $(8)$ is a path measure charging absolutely continuous paths. The constraint $\left(Q_{t}=\right.$ vol, $\left.\forall 0 \leq t \leq 1\right)$ reflects the volume preservation. The prescription that $Q_{01}=\pi$ varies among all the possible correlation structures between the initial and final positions with average profile $Q_{0}=Q_{1}=$ vol. It is a relaxation of $q_{1}(\cdot)=h$ as can be seen by taking $\pi(d x d y)=$ $\pi^{h}(d x d y):=\operatorname{vol}(d x) \delta_{h(x)}(d y)$.

It is proved in [Bre89b] that any $P \in \mathrm{P}(\Omega)$ such that $\left\{\begin{array}{l}P_{t}=\text { vol, } \forall t \text { and } P_{01}=\pi \\ \ddot{X}_{t}+\nabla p\left(t, X_{t}\right)=0, \forall t, P \text {-a.e. }\end{array}\right.$, for some pressure field $p$, solves the geodesic problem (8). Keeping Arnold's point of view, we see that the $\mathrm{P}\left(\mathcal{X}^{2}\right)$-valued flow $\left(P_{0 t}\right)_{0 \leq t \leq 1}$ is the generalized solution of Arnold's geodesic equation (7). In this approach one can recover the velocity field by defining a probability measure $\sigma$ on $[0,1] \times \mathcal{X} \times \mathbb{R}^{n}$,

$$
\int_{[0,1] \times \mathcal{X} \times \mathbb{R}^{n}} f(t, x, v) \sigma(d t d x d v):=E_{P} \int_{0}^{1} f\left(t, X_{t}, \dot{X}_{t}\right) d t
$$

This measure can be considered as a generalized velocity that solves the Euler equation in the sense of DiPerna and Majda, see [Bre99] for details.

Navier-Stokes equation. Navier-Stokes equation is a modification of the Euler equation where some viscosity term is added. Its Newtonian expression is

$$
\mathrm{D}_{t} v=a \Delta v-\nabla p
$$

where $\Delta v$, the Laplace operator applied to $v$, represents a viscosity force, $a>0$. This equation mixes the acceleration $\mathrm{D}_{t} v$ which is easily expressed in Lagrangian coordinates and the viscosity term $\Delta v$ which is easily expressed in terms of Eulerian coordinates. Rewriting everything in Eulerian terms leads to

$$
\begin{cases}\partial_{t} v+v \cdot \nabla v-a \Delta v+\nabla p=0, & t \geq 0, x \in \mathcal{X} \\ \nabla \cdot v=0, & t \geq 0, x \in \mathcal{X} \\ v(0, \cdot)=v_{0}, & t=0\end{cases}
$$

This is the Navier-Stokes equation of the unknown $(v, p)$ for an incompressible fluid seen as a Cauchy problem with a given initial velocity field $v_{0}$. 
Introducing the Brownian motion. The presence of the Laplacian in (9) strongly suggests that considering Brownian paths instead of regular paths in (8) might lead us to an approach of the Navier-Stokes equation similar to Brenier's approach to the Euler equation. One immediately faces a first problem: defining the kinetic action $\int_{[0,1]}\left|\dot{X}_{t}\right|^{2} / 2 d t$, since the Brownian sample paths are nowhere differentiable and any discrete approximation of the action diverges to infinity. One is forced to introduce an analogue of the average action $E_{Q} \int_{[0,1]}\left|\dot{X}_{t}\right|^{2} / 2 d t$ that appeared in (8) by considering

$$
E_{Q} \int_{[0,1]}\left|v_{t}^{Q}(X)\right|^{2} / 2 d t
$$

with a relevant notion of stochastic velocity $v_{t}^{Q}(X)$ introduced in place of the usual velocity $v\left(t, X_{t}\right)=\dot{X}_{t}$, undefined in the present context where the path measure $Q$ charges Brownian sample paths. A relevant notion of stochastic velocity was introduced by Nelson in [Nel67]. The forward stochastic velocity is defined by

$$
\vec{v}_{t}^{Q}\left(X_{[0, t]}\right):=\lim _{h \rightarrow 0^{+}} \frac{1}{h} E_{Q}\left(X_{t+h}-X_{t} \mid X_{[0, t]}\right)
$$

and its backward counterpart by

$$
\overleftarrow{v}_{t}^{Q}\left(X_{[t, 1]}\right):=\lim _{h \rightarrow 0^{+}} \frac{1}{h} E_{Q}\left(X_{t}-X_{t-h} \mid X_{[t, 1]}\right),
$$

provided that $X$ is a $Q$-integrable process and these limits exist in some sense. The stochastic action (10) computed with Nelson's stochastic velocity $v^{Q}=\vec{v}^{Q}$ can be expressed in terms of a relative entropy with respect to the reversible Brownian motion.

In the whole paper the reference path measure is the law $R$ of the reversible Brownian motion on $\mathcal{X}=\mathbb{T}^{n}$ or $\mathcal{X}=\mathbb{R}^{n}$ with constant diffusion coefficient $a>0$.

Definition 2.1. The reversible Brownian path measure $R$ is defined by

$$
R=\int_{\mathcal{X}} R^{x}(\cdot) \operatorname{vol}(d x) \in \Omega
$$

where for each $x \in \mathcal{X}, R^{x} \in \mathrm{P}(\Omega)$ is the law of $x+\sqrt{a} B$ where $B$ is a standard Brownian motion on $\mathcal{X}$ starting from 0 .

Roughly speaking, $R$ is the Wiener measure with diffusion coefficient $a$ on $\mathcal{X}$ starting from $R_{0}=$ vol $\in \mathrm{P}(\mathcal{X})$. As $R$ is a reversible Markov measure, its forward and backward velocities are opposite to each other: $\vec{v}^{R}+\overleftarrow{v}^{R}=0$. Be aware that we have chosen Nelson's convention when defining the backward velocity $\breve{v}_{t}^{Q}\left(X_{[t, 1]}\right)=-\left[\vec{v}_{1-t}^{\left(X^{*}\right)_{\#} Q} \circ X^{*}\right]\left(X_{[t, 1]}\right)$ where $X_{t}^{*}:=X_{1-t}, 0 \leq t \leq 1$, is the time reversed canonical process.

When $\mathcal{X}=\mathbb{R}^{n}, R \in \mathrm{M}(\Omega)$ is an unbounded $\sigma$-finite measure and when $\mathcal{X}=\mathbb{T}^{n}, R \in \mathrm{P}(\Omega)$ is a probability measure. Note that in any case, the conditioned path measures $R^{x} \in \mathrm{P}(\Omega)$ are probability measures.

Girsanov's theory allows to show that for any $Q \in \mathrm{M}(\Omega)$ with a finite relative entropy $H(Q \mid R)<\infty$, there is some predictable vector field $\vec{v}^{Q}\left(t, X_{[0, t]}\right)$ such that $Q$ is the unique solution, among the path measures with the initial marginal $Q_{0}$ and which are absolutely continuous with respect to $R$, of the martingale problem.

For every differentiable in time, twice differentiable in space function $f$ and all $0 \leq t \leq 1$,

$$
f\left(t, X_{t}\right)-f\left(0, X_{0}\right)-\int_{0}^{t} L_{s} f\left(s, X_{s}\right) d s
$$


is a martingale, where

$$
L_{t} u=\vec{v}^{Q}\left(t, X_{[0, t]}\right) \cdot \nabla u+a \Delta u / 2 .
$$

Moreover, we have

$$
H(Q \mid R)=H\left(Q_{0} \mid R_{0}\right)+\frac{1}{2 a} E_{Q} \int_{[0,1]}\left|\vec{v}^{Q}\left(t, X_{[0, t]}\right)\right|^{2} d t .
$$

For the details, see for instance [Léo12b].

These considerations were put forward a long time ago by Yasue in [Yas83] who introduced the stochastic action (10) but didn't take advantage of its representation (13) in terms of the relative entropy, although (13) is invoked in [Yas83, p. 135]. Since $R$ is reversible with $R_{0}=R_{1}=$ vol, we also obtain

$$
H(Q \mid R)=H\left(Q_{0} \mid \mathrm{vol}\right)+\frac{1}{2 a} E_{Q} \int_{[0,1]}\left|\vec{v}_{t}^{Q}\right|^{2} d t=\frac{1}{2 a} E_{Q} \int_{[0,1]}\left|\overleftarrow{v}_{t}^{Q}\right|^{2} d t+H\left(Q_{1} \mid \mathrm{vol}\right)
$$

where the stochastic drift fields $\vec{v}^{Q}$ and $\overleftarrow{v}^{Q}$ given by Girsanov's theory are precisely the forward and backward stochastic velocities of $Q$ properly defined in some $L^{2}$ spaces. It is therefore natural to address the following entropy minimization problem

$$
H(Q \mid R) \rightarrow \min ; \quad Q \in \mathrm{M}(\Omega):\left[Q_{t}=\mathrm{vol}, \forall 0 \leq t \leq 1\right], Q_{01}=\pi
$$

in analogy with Brenier's problem (8). Of course, as $Q_{0}$ and $Q_{1}$ are prescribed to be the volume measure, we see that

$$
H(Q \mid R)=\frac{1}{2 a} E_{Q} \int_{[0,1]}\left|\vec{v}_{t}^{Q}\right|^{2} d t=\frac{1}{2 a} E_{Q} \int_{[0,1]}\left|\overleftarrow{v}_{t}^{Q}\right|^{2} d t
$$

strengthening the analogy with (8).

The BS-problem. We propose and investigate a new hydrodynamic variational problem, aimed to model the dynamics of viscous flows. This problem is a generalization to the viscous case of Brenier relaxation of Arnold geodesic problem for incompressible nonviscous fluids.

In particular we are not dealing with Navier-Stokes equation, which is a Cauchy problem for the velocity. Instead, in a Lagrangian perspective, we are considering measures on path spaces of Lagrangian flows on one hand, and subject to boundary conditions on the other.

Let us remark the fact that Brenier introduced his relaxation problem in situations where classical solutions do not exist. The present model should be seen as an alternative point of view in the study of viscous hydrodynamics, specially when classical solutions of the Navier-Stokes theory may fail to exist.

However some links exist between solutions to Navier-Stokes equation and minimization of entropy, similar to the ones between classical solution to Euler equation and action minimization for generalized solutions in [Bre89a, Theorem 5.1]. The following Proposition is proved in $[\mathrm{ACF}]$.

Proposition 2.2. Let u be a regular solution on $[0, T]$ to the incompressible Navier-Stokes equation

$$
\frac{\partial}{\partial t} u(t, x)+\nabla u(t, x) \cdot u(t, x)-\nu \Delta u(t, x)=-\nabla p(T-t, x)
$$

on the torus $\mathbb{T}^{n}$. Assume that the pressure satisfies $\nabla^{2} p(t, x) \leq R \operatorname{Id}$ with $R T^{2} \leq \pi^{2}$. Consider the Brownian motion with drift $\hat{u}(t, x)=-u(T-t, x)$, satisfying

$$
d g_{t}=\sqrt{2 a} d B_{t}+\hat{u}\left(t, g_{t}\right) d t
$$


where $B_{t}$ is a Brownian motion in $\mathbb{R}^{n}$ and $g_{0}$ has uniform law in $\mathbb{T}^{n}$.

Then the law $Q$ of the process $g$ is a minimizer of $H(\cdot \mid R)$ in the class of laws of processes

$$
\mathcal{G}=\left\{g^{*}, d g_{t}^{*}=\sqrt{2 a} d B_{t}+D_{t} g^{*} d t, g_{0}^{*}=g_{0}, g_{T}^{*}=g_{T}\right\},
$$

$D_{t} g^{*}$ denoting the drift of $g^{*}$ in the considered filtration.

In $[\mathrm{ACF}]$ it is also proved that without any assumption on the pressure, the measure of the Brownian flow with velocity $\hat{u}$ is a critical point for a large class of perturbations of the flow.

Recall that the dynamical version of the Schrödinger problem amounts to minimize the relative entropy

$$
H(Q \mid R) \rightarrow \min ; \quad Q \in \mathrm{M}(\Omega): Q_{0}=\mu_{0}, Q_{1}=\mu_{1}
$$

among all the path measures $Q$ such that the initial and final marginals $Q_{0}$ and $Q_{1}$ are prescribed to be equal to given measures $\mu_{0}$ and $\mu_{1} \in \mathrm{M}(\mathcal{X})$. For more details on this convex optimization problem see [Föl88, FG97, Léo14b]. As Problem (14) is an hybrid of Brenier's problem (8) and Schrödinger's problem (15), we call it the Brenier-Schrödinger problem. We introduce the following extension of (15):

$$
H(Q \mid R) \rightarrow \min ; \quad Q \in \mathrm{M}(\Omega):\left(Q_{t}=\mu_{t}, \forall t \in \mathcal{T}\right), \quad Q_{01}=\pi
$$

with $\mathcal{T}$ a measurable subset of $[0,1]$ and $\left(\mu_{t}\right)_{t \in \mathcal{T}}$ a prescribed set of nonnegative measures on $\mathcal{X}$. It is a slight extension of (14) where the state space $\mathcal{X}$ and the prescribed marginals $\left(\mu_{t}\right)_{t \in \mathcal{T}}$ are general. We still call the extension (BS) of (14) the BS-problem.

Of course, for this problem to admit a solution, it is necessary that $H\left(\mu_{t} \mid \operatorname{vol}\right)<\infty$ for all $t \in \mathcal{T}, H\left(\pi \mid R_{01}\right)<\infty$ (recall (3)) and $\begin{cases}\pi_{0} & :=\pi(\cdot \times \mathcal{X})=\mu_{0}, \\ \pi_{1} & :=\pi(\mathcal{X} \times \cdot)=\mu_{1} .\end{cases}$

Proposition 2.3. Problem (BS) admits a solution if and only if there exists some $Q \in$ $\mathrm{M}(\Omega)$ such that $Q_{t}=\mu_{t}$ for all $t \in \mathcal{T}, Q_{01}=\pi$ and $H(Q \mid R)<\infty$. In this case, the solution $P$ is unique.

Remark 2.4. It can be checked without difficulty that this result is also valid when the state space $\mathcal{X}$ is a stochastically complete Riemannian manifold with smooth boundary, $R$ is the reversible Brownian path measure with initial marginal $R_{0}=$ vol.

Fundamental example on the torus. As a basic important example, we take $R \in \mathrm{P}(\Omega)$ the Wiener measure on the flat torus $\mathcal{X}=\mathbb{T}^{n}$ with initial marginal $R_{0}=$ vol : the normalized volume measure (so that $R$ is reversible), $\mu_{t}=$ vol for all $t \in \mathcal{T}=[0,1]$ and $\pi$ any bi-stochastic measure, i.e. such that $\pi_{0}=\pi_{1}=$ vol. In this setting, (BS) becomes (14) which is as close as possible to Brenier's problem (8).

It is proved in Corollary 6.2 that in this precise setting, the BS-problem (14) admits a solution if and only if $\pi$ is such that $H\left(\pi \mid R_{01}\right)<\infty$.

Remark 2.5. In the context of Remark 2.4, the fundamental example extends to a compact manifold $\mathcal{X}$ on which acts transitively a compact group of isometries with bi-invariant metric.

A simplified problem. It will be convenient to consider the easy version of the BSproblem (BS) with a finite set $\mathcal{T}=\left\{t_{1}, \ldots, t_{K}\right\}$ :

$$
H(Q \mid R) \rightarrow \min ; \quad Q \in \mathrm{M}(\Omega):\left(Q_{t_{k}}=\mu_{t_{k}}, 0<t_{1}<\cdots<t_{K}<1\right), \quad Q_{01}=\pi
$$

where only a finite number of marginal constraints are prescribed. 


\section{Duality}

The duality of Brenier's problem was studied in [Bre93]. The present section is devoted to the dual of the BS-problem. In contrast with Brenier's problem which is affine, the strict convexity of the BS-problem allows for a rather standard treatment based on general convex analysis in infinite dimensional spaces stated below at Theorem 3.4. The main results of the section are the dual equality of Proposition 3.3 holding under weak hypotheses, and a characterization of the solution of the BS-problem stated at Corollary 3.7, valid under restrictive regularity assumptions.

The dual problem of $(\mathrm{BS})$ is stated at (19). Its unknown are a pressure scalar field $p$ : $[0,1] \times \mathcal{X} \rightarrow \mathbb{R}$ in duality with the incompressibility constraint and a function $\eta: \mathcal{X}^{2} \rightarrow \mathbb{R}$ in duality with the endpoint constraint $\pi$.

Dual equality. Let us denote for all $x \in \mathcal{X}, R^{x}:=R\left(\cdot \mid X_{0}=x\right) \in \mathrm{P}(\Omega)$ and $R^{\mu_{0}}=$ $\int_{\mathcal{X}} R^{x}(\cdot) \mu_{0}(d x) \in \mathrm{M}(\Omega)$. They describe respectively the reference kinematics starting from $x$ or from the initial distribution $R_{0}^{\mu_{0}}=\mu_{0}$. By (2) with $f=X_{0}$,

$$
H(Q \mid R)=H\left(\mu_{0} \mid R_{0}\right)+H\left(Q \mid R^{\mu_{0}}\right)
$$

for all $Q \in \mathrm{M}(\Omega)$ such that $Q_{0}=\mu_{0}$. Therefore, as soon as $H\left(\mu_{0} \mid R_{0}\right)<\infty$ (this is verified when (BS) admits a solution), it is equivalent to solve the modified BS-problem

$$
H\left(Q \mid R^{\mu_{0}}\right) \rightarrow \min ; \quad Q \in \mathrm{M}(\Omega): Q_{t}=\mu_{t}, t \in \mathcal{T}, \quad Q_{01}=\pi,
$$

or $(\mathrm{BS})$. The problems $(\mathrm{BS})$ and $\left(\mathrm{BS}^{\mu_{0}}\right)$ share the same solution but their values differ from the quantity $H\left(\mu_{0} \mid R_{0}\right)$ that only depends on the prescribed data $R$ and $\mu$. As far as duality is concerned, it will be a little bit more comfortable to consider $\left(\mathrm{BS}^{\mu_{0}}\right)$ rather than (BS).

Take $\alpha$ a probability measure on $\mathcal{T}$ and consider the following weakening of $(\mathrm{BS})$ :

$$
H(P \mid R) \rightarrow \min ; \quad P \in \mathrm{M}(\Omega):\left(P_{t}=\mu_{t}, \quad \text { for } \alpha \text {-almost all } t \in \mathcal{T}\right), \quad Q_{01}=\pi .
$$

For instance, one may take $\alpha=K^{-1} \sum_{1 \leq k \leq K} \delta_{t_{k}}$ for the BS-problem (16). Choosing $\alpha=\operatorname{Leb}_{[0,1]}$ leads to $Q_{t}=\mu_{t}$, for almost all $t \in[0,1]$ which is a slight weakening of $Q_{t}=\mu_{t}, \forall t \in[0,1]$ in the original BS-problem (14). Nevertheless, the following result holds.

Lemma 3.1. Assume that $\alpha$ has a full support, i.e. $\operatorname{supp} \alpha=\mathcal{T}$, and consider the following statements:

(i) (BS) admits a solution;

(ii) $t \in \mathcal{T} \mapsto \mu_{t} \in \mathrm{P}(\mathcal{X})$ is weakly continuous on $\mathcal{T}$;

(iii) (BS) is equivalent to (18).

We have: $(i) \Longrightarrow($ ii $) \Longrightarrow($ iii $)$.

Proof. Since for any $P \in \mathrm{M}(\Omega), t \in \mathcal{T} \mapsto P_{t} \in \mathrm{P}(\mathcal{X})$ is weakly continuous (this follows from the continuity of the sample paths), it is necessary for (BS) to admit a solution such that $t \mapsto \mu_{t}$ is also weakly continuous. In such a case, under the assumption that $\operatorname{supp} \alpha=$ $\mathcal{T}$, the constraint $\left(P_{t}=\mu_{t}\right.$, for $\alpha$-almost all $\left.t \in \mathcal{T}\right)$ is equivalent to $\left(P_{t}=\mu_{t}, \forall t \in \mathcal{T}\right)$.

For the moment, it is assumed that the constraint $\mu=\left(\mu_{t}\right)_{t \in \mathcal{T}}$ is a flow of probability measures and $\pi \in \mathrm{P}\left(\mathcal{X}^{2}\right)$ is also a probability measure. In particular, this implies that $(\mathrm{BS})$ is

$$
H(Q \mid R) \rightarrow \min ; \quad Q \in \mathrm{P}(\Omega):\left(Q_{t}=\mu_{t}, \forall t \in \mathcal{T}\right), \quad Q_{01}=\pi
$$

where $Q$ lives in $\mathrm{P}(\Omega)$ rather than in $\mathrm{M}(\Omega)$. 
Hypotheses 3.2. (a) The constraint $\mu=\left(\mu_{t}\right)_{t \in \mathcal{T}}$ is a flow of probability measures.

(b) The mapping $t \in \mathcal{T} \mapsto \mu_{t} \in \mathrm{P}(\mathcal{X})$ is weakly continuous on $\mathcal{T}$.

(c) We choose $\alpha \in \mathrm{P}(\mathcal{T})$ such that $\operatorname{supp} \alpha=\mathcal{T}$.

It follows from Lemma 3.1 that we can assume Hypothesis 3.2-(b) almost without any loss of generality, so that (BS) and (18) are equivalent.

The aim of this section is to prove a dual equality for (BS).

Before stating it at Proposition 3.3, we must introduce some notions and notation. The value function of problem $\left(\mathrm{BS}^{\mu_{0}}\right)$ is denoted by

$$
J(\mu, \pi):=\inf \left(\mathrm{BS}^{\mu_{0}}\right)=\inf \left\{H\left(Q \mid R^{\mu_{0}}\right) ; Q \in \mathrm{P}(\Omega): Q_{t}=\mu_{t}, \forall t \in \mathcal{T}, Q_{01}=\pi\right\} .
$$

We denote respectively $B(\mathcal{T} \times \mathcal{X})$ and $B\left(\mathcal{X}^{2}\right)$ the spaces of bounded measurable functions of $\mathcal{T} \times \mathcal{X}$ and $\mathcal{X}^{2}$.

Proposition 3.3. For any $\pi \in \mathrm{P}\left(\mathcal{X}^{2}\right), \mu \in \mathrm{P}(\mathcal{X})^{\mathcal{T}}$ and $\alpha \in \mathrm{P}(\mathcal{T})$ satisfying the Hypotheses 3.2, we have

$$
\inf (\mathrm{BS})=H\left(\mu_{0} \mid R_{0}\right)+J(\mu, \pi) .
$$

Moreover, for any class of functions $\mathcal{A}$ dense in $B(\mathcal{T} \times \mathcal{X}) \times B\left(\mathcal{X}^{2}\right)$ with respect to the pointwise convergence, the dual equality is

$$
\begin{aligned}
J(\mu, \pi)=\sup _{(p, \eta) \in \mathcal{A}}\left\{\left\langle p, \mu^{\alpha}\right\rangle+\langle\eta, \pi\rangle\right. & \\
& \left.-\int_{\mathcal{X}} \log E_{R^{x}} \exp \left(\int_{\mathcal{T}} p\left(t, X_{t}\right) \alpha(d t)+\eta\left(x, X_{1}\right)\right) \mu_{0}(d x)\right\},
\end{aligned}
$$

where we denote $\mu^{\alpha}(d t d x):=\alpha(d t) \mu_{t}(d x)$.

Of course, the first identity is a direct consequence of (17).

The remainder of the present section is devoted to the proof of the dual equality.

An abstract duality result. We begin stating an already known abstract result about convex duality. We shall apply it later to derive the dual problem of (BS) and some basic related relations. In these lines, we rely on general results about convex duality as presented for instance in the lecture notes [Léo]. Let $U$ and $V$ be two Hausdorff locally convex topological vector spaces with respective topological dual spaces $U^{\prime}$ and $V^{\prime}$. Let us consider the following minimization problem

$$
I(\ell) \rightarrow \min ; \quad \ell \in U^{\prime}: T \ell=k_{o}
$$

where $I$ is a convex $(-\infty, \infty]$-valued function on $U^{\prime}, T: U^{\prime} \rightarrow V^{\prime}$ is a linear operator and $k_{o} \in V^{\prime}$. We assume that the algebraic adjoint operator $T^{*}$ of $T$ satisfies $T^{*}(V) \subset U$, so that one can write $\left[T^{*} v\right](\ell)=\left\langle T^{*} v, \ell\right\rangle_{U, U^{\prime}}=\langle v, T \ell\rangle_{V, V^{\prime}}$. It follows that the diagram

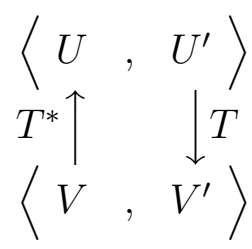

is meaningful. The associated dual problem is

$$
\left\langle v, k_{o}\right\rangle-I^{*}\left(T^{*} v\right) \rightarrow \max ; \quad v \in V
$$

where

$$
I^{*}(u):=\sup _{\ell \in U^{\prime}}\{\langle\ell, u\rangle-I(\ell)\}, \quad u \in U
$$


is the convex conjugate of $I$ with respect to the duality $\left\langle U, U^{\prime}\right\rangle$.

Theorem 3.4. Let us suppose that the following assumptions on $I$ and $T$ hold:

(i) $I$ is a convex $\sigma\left(U^{\prime}, U\right)$-lower semicontinuous function such that inf $I>-\infty$;

(ii) there exists an open neighbourhood $N$ of 0 in $U$ such that $\sup _{u \in N} I^{*}(u)<+\infty$;

(iii) $T^{*} V \subset U$.

Then, the following assertions are verified.

(1) If $\inf (\mathcal{P})<+\infty$, the primal problem $(\mathcal{P})$ admits at least a solution and if $I$ is strictly convex, this solution is unique.

(2) The dual equality $\inf (\mathcal{P})=\sup (\mathcal{D})$ holds. That is

$$
\inf \left\{I(\ell) ; \ell \in U^{\prime}: T \ell=k_{o}\right\}=\sup _{v \in V}\left\{\left\langle k_{o}, v\right\rangle-I^{*}\left(T^{*} v\right)\right\} \in(-\infty,+\infty] .
$$

(3) The primal and dual problems are attained at $\bar{\ell}$ and $\bar{v}$ respectively if and only if the following relations hold: $\left\{\begin{array}{l}T \bar{\ell}=k_{o}, \\ \bar{\ell} \in \partial I^{*}\left(T^{*} \bar{v}\right),\end{array}\right.$ where

$\partial I^{*}\left(T^{*} \bar{v}\right):=\left\{\ell \in U^{\prime} ; I^{*}\left(T^{*} \bar{v}+w\right) \geq I^{*}\left(T^{*} \bar{v}\right)+\langle\ell, w\rangle_{U^{\prime}, U}, \forall w \in U\right\}$ denotes the subdifferential of $I^{*}$ at $T^{*} \bar{v}$.

Proof of Proposition 3.3. We apply Theorem 3.4 to the primal problem (BS). We first chose a relevant set of vector spaces and objective functions $U, U^{*}, I$ and $I^{*}$. Then, we look at the constraint operators $T$ and $T^{*}$. Finally, Theorem 3.4 is applied in this setting.

The objective functions $I$ and $I^{*}$. Let us denote $\mathrm{B}(\Omega)$ the space of all bounded measurable functions on $\Omega$ and equip it with the uniform norm $\|u\|:=\sup _{\omega \in \Omega}|u(\omega)|, u \in \mathrm{B}(\Omega)$. Its topological dual space is denoted by $\mathrm{B}(\Omega)^{\prime}$. The convex function

$$
\Theta(u):=\int_{\mathcal{X}} \log \left(E_{R^{x}} e^{u}\right) \mu_{0}(d x), \quad u \in \mathrm{B}(\Omega)
$$

is well defined on $\mathrm{B}(\Omega)$ because for any $u \in \mathrm{B}(\Omega)$

$$
I \log E_{R^{x}} e^{u} \mid \leq\|u\|, \quad \forall x \in \mathcal{X},
$$

implying

$$
|\Theta(u)| \leq\|u\|<\infty .
$$

Comparing $(\mathrm{BS})$ with $(\mathcal{P})$ and taking Lemma 3.5 below into account, we see that a good framework to work with is: $(U,\|\cdot\|)=(\mathrm{B}(\Omega),\|\cdot\|)$ and

$$
I(Q):=\Theta^{*}(Q)=\sup _{u \in \mathrm{B}(\Omega)}\left\{\langle Q, u\rangle-\int_{\mathcal{X}} \log \left(E_{R^{x}} e^{u}\right) \mu_{0}(d x)\right\}, \quad Q \in \mathrm{B}(\Omega)^{\prime},
$$

the convex conjugate of $\Theta$.

Lemma 3.5. For any $Q \in \mathrm{B}(\Omega)^{\prime}, I(Q)= \begin{cases}H\left(Q \mid R^{\mu_{0}}\right), & \text { if } Q \in \mathrm{P}(\Omega) \text { and } Q_{0}=\mu_{0}, \\ +\infty, & \text { otherwise. }\end{cases}$

Proof. This proof follows the line of the proof of [Léo12a, Lemma 4.2].

Let $Q \in \mathrm{B}(\Omega)^{\prime}$ be such that $I(Q)<\infty$.

(a) Let us show that $Q \geq 0$. Take $u \in \mathrm{B}(\Omega), u \geq 0$. As for all $a \leq 0, \Theta(a u) \leq 0$, we get

$$
I(Q) \geq \sup _{a \leq 0}\{a\langle Q, u\rangle-\Theta(a u)\} \geq \sup _{a \leq 0} a\langle Q, u\rangle= \begin{cases}0, & \text { if }\langle Q, u\rangle \geq 0 \\ +\infty, & \text { otherwise. }\end{cases}
$$

Hence, $I(Q)<\infty$ implies that $\langle Q, u\rangle \geq 0$, for all $u \geq 0$, which is the desired result. 
(b) Let us show that $Q$ is a positive measure. For a positive element of $\mathrm{B}(\Omega)^{\prime}$ to be a measure it is sufficient (and necessary) that it is $\sigma$-additive. This means that for any decreasing sequence $\left(u_{n}\right)_{n \geq 0}$ of measurable bounded functions such that $\lim _{n \geq 0} u_{n}(\omega)=0$ for all $\omega \in \Omega$, we have

$$
\lim _{n \rightarrow \infty}\left\langle Q, u_{n}\right\rangle=0
$$

Let $\left(u_{n}\right)_{n \geq 0}$ be such a sequence. By dominated convergence, for all $a \geq 0$, we obtain $\lim _{n \rightarrow \infty} \Theta\left(a u_{n}\right)=0$. Therefore,

$$
I(Q) \geq \sup _{a \geq 0} \limsup _{n \rightarrow \infty}\left\{a\left\langle Q, u_{n}\right\rangle-\Theta\left(a u_{n}\right)\right\}=\sup _{a \geq 0} a \limsup _{n \rightarrow \infty}\left\langle Q, u_{n}\right\rangle
$$

and $I(Q)<\infty$ implies that $\lim _{\sup _{n \rightarrow \infty}}\left\langle Q, u_{n}\right\rangle \leq 0$. Since, we already know that $Q \geq 0$, this gives the desired result: $\lim _{n \rightarrow \infty}\left\langle Q, u_{n}\right\rangle=0$.

(c) Let $Q \in \mathrm{M}(\Omega)$. Taking $u=f\left(X_{0}\right)$ in $\sup _{u}$ gives

$$
I(Q) \geq \sup _{f}\left\langle f, Q_{0}-\mu_{0}\right\rangle= \begin{cases}0, & \text { if } Q_{0}=\mu_{0} \\ \infty, & \text { otherwise }\end{cases}
$$

Hence, $I(Q)<\infty$ implies that $Q_{0}=\mu_{0} \in \mathrm{P}(\mathcal{X})$ which in turns implies that $Q$ is also a probability measure.

In this case, $Q=\int_{\mathcal{X}} Q^{x}(\cdot) \mu_{0}(d x)$ and

$$
\begin{aligned}
I(Q) & =\sup _{u} \int_{\mathcal{X}}\left(E_{Q^{x}} u-\log E_{R^{x}} e^{u}\right) \mu_{0}(d x) \\
& \left.\stackrel{(i)}{=} \sup _{k \geq 1} \int_{\mathcal{X} u^{x}:\left|u^{x}\right| \leq k} \sup _{Q^{x}} u^{x}-\log E_{R^{x}} e^{u^{x}}\right) \mu_{0}(d x) \\
& =\sup _{k \geq 1} \int_{\mathcal{X}} \sup _{v:|v| \leq k}\left(E_{Q^{x}} v-\log E_{R^{x}} e^{v}\right) \mu_{0}(d x) \\
& \stackrel{(i i)}{=} \int_{\mathcal{X}} H\left(Q^{x} \mid R^{x}\right) \mu_{0}(d x) \\
& \stackrel{(17)}{=} H\left(Q \mid R^{\mu_{0}}\right)
\end{aligned}
$$

which is the announced result. Let us give some precisions about this series of identities. At (i), we used the notation $u^{x}$ for the restriction of $u$ to $\Omega^{x}:=\left\{X_{0}=x\right\} \subset \Omega$. Note that the inversion of $\sup _{u}$ and $\int_{\mathcal{X}}$ is valid since any function $u \in \mathrm{B}(\Omega)$ can be identified with a measurable kernel $\left(u^{x} \in B\left(\Omega^{x}\right), x \in \mathcal{X}\right)$ by $u=u^{X_{0}}$. Identity (ii) follows from a standard variational representation of the relative entropy of probability measures (see [GL10, Appendix] for instance) combined with Beppo-Levi's monotone convergence theorem.

This completes the proof of the lemma.

Let us compute $I^{*}=\Theta^{* *}$ defined by $I^{*}(u):=\sup _{Q \in \mathrm{B}(\Omega)^{\prime}}\{\langle Q, u\rangle-I(Q)\}, u \in \mathrm{B}(\Omega)$. As $\Theta$ is convex (by Hölder's inequality) and lower $\sigma\left(\mathrm{B}(\Omega), \mathrm{B}(\Omega)^{\prime}\right)$-semicontinuous (by Fatou's lemma, it is lower $\|\cdot\|$-semicontinuous and since it is convex, it turns out to be weakly semicontinuous), it is equal to its convex biconjugate. This means that

$$
I^{*}=\Theta^{* *}=\Theta .
$$


The constraint operators $T$ and $T^{*}$. For any $Q \in \mathrm{B}(\Omega)^{\prime}$, we define $\widetilde{Q} \in B(\mathcal{T} \times \mathcal{X})^{\prime}$ by

$$
\langle\widetilde{Q}, p\rangle=\int_{\mathcal{T}}\left\langle Q, p\left(t, X_{t}\right)\right\rangle \alpha(d t), \quad p \in B(\mathcal{T} \times \mathcal{X})
$$

Clearly, when $Q$ belongs to $\mathrm{P}(\Omega), \widetilde{Q}$ is the measure defined by $\widetilde{Q}(d t d x)=\alpha(d t) Q_{t}(d x)$. Hence, defining $\mu^{\alpha}(d t d x):=\mu_{t}(d x) \alpha(d t)$, we see that $\widetilde{Q}=\mu^{\alpha}$ is equivalent to $Q_{t}=\mu_{t}$ for $\alpha$-almost all $t \in \mathcal{T}$.

For any $Q \in \mathrm{B}(\Omega)^{\prime}$, we define $Q_{01} \in B\left(\mathcal{X}^{2}\right)^{\prime}$ by: $\left\langle Q_{01}, \eta\right\rangle=\left\langle Q, \eta\left(X_{0}, X_{1}\right)\right\rangle, \forall \eta \in B\left(\mathcal{X}^{2}\right)$. Putting everything together, the constraint operator is defined by

$$
T Q:=\left(\widetilde{Q}, Q_{01}\right) \in B(\mathcal{T} \times \mathcal{X})^{\prime} \times B\left(\mathcal{X}^{2}\right)^{\prime}, \quad Q \in \mathrm{B}(\Omega)^{\prime}
$$

and the full constraint of (18) writes as

$$
T Q=\left(\mu^{\alpha}, \pi\right), \quad Q \in \mathrm{B}(\Omega)^{\prime} .
$$

It is time to identify the topological space $V$ as $V=B(\mathcal{T} \times \mathcal{X}) \times B\left(\mathcal{X}^{2}\right)$ equipped with the uniform norm $\|\cdot\|_{\mathcal{T} \times \mathcal{X}} \oplus\|\cdot\|_{\mathcal{X}^{2}}$, so that its topological space $V^{\prime}=B(\mathcal{T} \times \mathcal{X})^{\prime} \times B\left(\mathcal{X}^{2}\right)^{\prime}$ contains $\mathrm{M}_{b}(\mathcal{T} \times \mathcal{X}) \times \mathrm{M}_{b}\left(\mathcal{X}^{2}\right)$.

Let us compute the adjoint $T^{*}$ of $T$. For any $Q \in \mathrm{B}(\Omega)^{\prime}, p \in B(\mathcal{T} \times \mathcal{X})$ and $\eta \in B\left(\mathcal{X}^{2}\right)$,

$$
\left\langle Q, T^{*}(p, \eta)\right\rangle=\langle T Q,(p, \eta)\rangle=\langle\widetilde{Q}, p\rangle+\left\langle Q_{1}, \eta\right\rangle=\left\langle Q, \int_{\mathcal{T}} p\left(t, X_{t}\right) \alpha(d t)+\eta\left(X_{0}, X_{1}\right)\right\rangle .
$$

Consequently, we see that

$$
T^{*}(p, \eta)=\int_{\mathcal{T}} p\left(t, X_{t}\right) \alpha(d t)+\eta\left(X_{0}, X_{1}\right)
$$

The dual problem. We gathered all the ingredients to see that the dual problem of (18) is

$$
\begin{gathered}
\int_{\mathcal{T} \times \mathcal{X}} p d \mu^{\alpha}+\int_{\mathcal{X}^{2}} \eta d \pi-\int_{\mathcal{X}} \log E_{R^{x}} \exp \left(\int_{\mathcal{T}} p\left(t, X_{t}\right) \alpha(d t)+\eta\left(x, X_{1}\right)\right) \mu_{0}(d x) \rightarrow \max \\
p \in B(\mathcal{T} \times \mathcal{X}), \eta \in B\left(\mathcal{X}^{2}\right)
\end{gathered}
$$

Proof of Proposition 3.3. It remains to verify the assumptions of Theorem 3.4 to obtain

$$
\begin{aligned}
J(\mu, \pi)=\sup _{(p, \eta) \in B(\mathcal{T} \times \mathcal{X}) \times B\left(\mathcal{X}^{2}\right)}\{ & \left\langle p, \mu^{\alpha}\right\rangle+\langle\eta, \pi\rangle \\
& \left.-\int_{\mathcal{X}} \log E_{R^{x}} \exp \left(\int_{\mathcal{T}} p\left(t, X_{t}\right) \alpha(d t)+\eta\left(x, X_{1}\right)\right) \mu_{0}(d x)\right\} .
\end{aligned}
$$

(i) Being a convex conjugate, $I=\Theta^{*}$ is convex and lower semicontinuous with respect to $\sigma\left(\mathrm{B}(\Omega)^{\prime}, \mathrm{B}(\Omega)\right)$.

(ii) We see with $(22)$, that the function $I^{*}=\Theta$ is such that on the unit ball $N=$ $\{u \in \mathrm{B}(\Omega) ;\|u\| \leq 1\}$, we have: $\sup _{u:\|u\| \leq 1} \Theta(u)<\infty$.

(iii) It is clear that for any $p$ in $B(\mathcal{T} \times \mathcal{X})$ and $\eta$ in $B\left(\mathcal{X}^{2}\right), T^{*}(p, \eta)=\int_{\mathcal{T}} p\left(t, X_{t}\right) \alpha(d t)+$ $\eta\left(X_{0}, X_{1}\right)$ is in $\mathrm{B}(\Omega)$.

So far we have proved Proposition 3.3 in the special case where $\mathcal{A}=B(\mathcal{T} \times \mathcal{X}) \times B\left(\mathcal{X}^{2}\right)$. The extension to the case where $\mathcal{A}$ is pointwise dense in $B(\mathcal{T} \times \mathcal{X}) \times B\left(\mathcal{X}^{2}\right)$ follows easily from an approximation argument combined with the dominated convergence theorem using (21). This completes the proof of Proposition 3.3.

Remark 3.6. We have chosen a strong topology on $U=\mathrm{B}(\Omega)$ to insure the estimate $\sup _{u \in N} \Theta(u)<\infty$ at (ii) above. This explains why Lemma 3.5 is needed. 
Regular solutions. As a byproduct of this proof, Theorem 3.4 leaves us with the following

Corollary 3.7. Assume that Hypothesis 3.2 holds.

(1) If inf $(\mathrm{BS})<+\infty$, the primal problem (BS) admits a unique solution.

(2) Let $P \in \mathrm{P}(\Omega), p \in B(\mathcal{T} \times \mathcal{X})$ and $\eta \in B\left(\mathcal{X}^{2}\right)$. Both the primal problem (BS) and the dual problem (24) are attained respectively at $P$ and $(p, \eta)$ if and only if the constraints $P_{t}=\mu_{t}, \forall t \in \mathcal{T}$ and $P_{01}=\pi$ are satisfied and

$$
P=\frac{d \mu_{0}}{d R_{0}}\left(X_{0}\right) \exp \left(\eta\left(X_{0}, X_{1}\right)-\mathcal{Q}(p, \eta)\left(X_{0}\right)+\int_{\mathcal{T}} p\left(t, X_{t}\right) \alpha(d t)\right) R
$$

where

$$
\mathcal{Q}(p, \eta)(x):=\log E_{R^{x}} \exp \left(\int_{\mathcal{T}} p\left(t, X_{t}\right) \alpha(d t)+\eta\left(x, X_{1}\right)\right), \quad x \in \mathcal{X}, R_{0} \text {-a.s. }
$$

Proof. Statement (1) is a direct application of Theorem 3.4-(1) and the only thing to be checked for (2) is the computation of the subdifferential $\partial I^{*}\left(T^{*} \bar{v}\right)$ of Theorem 3.4-(3) in the present setting. With $I^{*}=\Theta$ given at (20), we obtain for any $u \in \mathrm{B}(\Omega)$,

$$
\begin{aligned}
\Theta^{\prime}(u) & =e^{u} \int_{\mathcal{X}} R^{x}(\cdot) \frac{\mu_{0}(d x)}{E_{R^{x}} e^{u}}=\exp \left(u-\log E_{R^{x_{0}}} e^{u}\right) \int_{\mathcal{X}} R^{x}(\cdot) \mu_{0}(d x) \\
& =\exp \left(u-\log E_{R^{x_{0}}} e^{u}\right) R^{\mu_{0}}=\frac{d \mu_{0}}{d R_{0}}\left(X_{0}\right) \exp \left(u-\log E_{R^{x_{0}}} e^{u}\right) R .
\end{aligned}
$$

We conclude with $(23)$, i.e. $T^{*} \bar{v}=T^{*}(p, \eta)=\int_{\mathcal{T}} p\left(t, X_{t}\right) \alpha(d t)+\eta\left(X_{0}, X_{1}\right)$.

\section{General shape of the solution}

Assuming the dual attainment $p \in B(\mathcal{T} \times \mathcal{X})$ and $\eta \in B\left(\mathcal{X}^{2}\right)$ as in Corollary 3.7-(2) is very restrictive. In general, even if the solution $P$ writes as $(25), p$ and $\eta$ might be unbounded or even might take the value $-\infty$ at some places.

It is proved in [BL] that, provided that the reference path measure $R$ is Markov, the solution $P$ to the BS-problem is a reciprocal path measure. A representation of the Radon-Nikodym derivative $d P / d R$ is also obtained. In order to state these results below at Theorem 4.7, it is necessary to recall the definitions of Markov and reciprocal measures and also of additive functional.

First of all, we need to make precise what a conditionable path measure is.

Definitions 4.1 (Conditionable path measure).

(1) A positive measure $Q \in \mathrm{M}(\Omega)$ is called a path measure.

(2) The path measure $Q \in \mathrm{M}(\Omega)$ is said to be conditionable if for all $t \in[0,1]$, $Q_{t}$ is a $\sigma$-finite measure on $\mathcal{X}$.

It is shown in [Léo14a] that for any conditionable path measure $Q \in \mathrm{M}(\Omega)$, the conditional expectation $E_{Q}\left(\cdot \mid X_{\mathcal{T}}\right)$ is well-defined for any $\mathcal{T} \subset[0,1]$. This is the reason for this definition.

Definition 4.2 (Markov measure). A path measure $Q$ on $\Omega$ is said to be Markov if it is conditionable and if for any $t \in[0,1]$ and for any events $A \in \sigma\left(X_{[0, t]}\right), B \in \sigma\left(X_{[t, 1]}\right)$

$$
Q\left(A \cap B \mid X_{t}\right)=Q\left(A \mid X_{t}\right) Q\left(B \mid X_{t}\right), \quad Q \text {-a.e. }
$$

This means that, knowing the present state $X_{t}$, the future and past informations $\sigma\left(X_{[t, 1]}\right)$ and $\sigma\left(X_{[0, t]}\right)$, are $Q$-independent. 


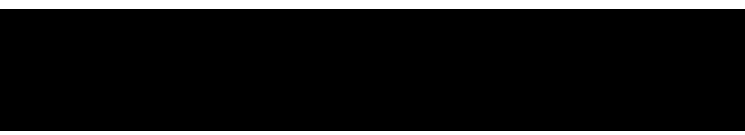

FigURE 1 .

Definition 4.3 (Reciprocal measure). A path measure $Q$ on $\Omega$ is called reciprocal if it is conditionable and for any times $0 \leq s \leq u \leq 1$ and any events $A \in \sigma\left(X_{[0, s]}\right), B \in$ $\sigma\left(X_{[s, u]}\right), C \in \sigma\left(X_{[u, 1]}\right)$,

see Figure 1,

$$
Q\left(A \cap B \cap C \mid X_{s}, X_{u}\right)=Q\left(A \cap C \mid X_{s}, X_{u}\right) Q\left(B \mid X_{s}, X_{u}\right) \quad \text { Q-a.e. }
$$

This property states that under $Q$, given the knowledge of the canonical process at both times $s$ and $u$, the events "inside" $[s, u]$ and those "outside" $(s, u)$ are conditionally independent. It is clearly time-symmetric.

Remarks 4.4. We recall basic relations between the Markov and reciprocal properties.

(a) Any Markov measure is reciprocal, but there are reciprocal measures that are not Markov.

(b) For any reciprocal measure $Q$, the conditional path measures $Q\left(\cdot \mid X_{0}\right)$ and $Q\left(\cdot \mid X_{1}\right)$ are Markov, $Q$-a.e.

Definition 4.5 (Additive functional). A measurable function $A_{[0,1]}: \Omega \rightarrow[-\infty, \infty)$ is said to an $R$-additive functional if for any finite partition $\bigsqcup_{k} I_{k}=[0,1]$ of the time interval $[0,1]$ with intervals $I_{k}$, we have

$$
A_{[0,1]}=\sum_{k} A_{I_{k}}, \quad R \text {-a.e. }
$$

where for all $k, A_{I_{k}}=A_{k}\left(X_{I_{k}}\right)$ is $\sigma\left(X_{I_{k}}\right)$-measurable.

With some abuse of notation, we shall write: $A_{[0,1]}=A=A\left(X_{[0,1]}\right)=\sum_{k} A\left(X_{I_{k}}\right)$.

We are now ready to state Theorem 4.7 and its hypotheses.

Assumption 4.6. (Strong irreducibility). The reference measure $R$ is Markov and it admits a transition density $r$ defined by for all $0 \leq s<t \leq 1$ by

$$
R\left(X_{t} \in d y \mid X_{s}=x\right):=r(s, x ; t, y) R_{t}(d y), \quad \forall x \in \mathcal{X}, R_{s} \text {-a.e. }
$$

which is positive in the sense that

$$
r(s, x ; t, y)>0, \quad \forall(x, y), R_{s} \otimes R_{t} \text {-a.e. }, \quad \forall 0 \leq s<t \leq 1 .
$$

Theorem 4.7. Under the above Assumptions 4.6, the following assertions hold true.

(a) The solution $P$ of $(\mathrm{BS})$, if it exists, is reciprocal and

$$
P=\exp \left(A\left(X_{\mathcal{T}}\right)+\eta\left(X_{0}, X_{1}\right)\right) R
$$

for some $[-\infty, \infty)$-valued $\sigma\left(X_{\mathcal{T}}\right)$-measurable additive functional $A\left(X_{\mathcal{T}}\right)$ and some measurable function $\eta: \mathcal{X}^{2} \rightarrow[-\infty, \infty)$, with the convention that $\exp (-\infty)=0$.

(b) We consider the prescribed marginals $\mu_{t_{k}} \in \mathrm{P}(\mathcal{X}), 1 \leq k \leq K$ at times $0 \leq t_{1}<$ $\cdots t_{K} \leq 1$ and the prescribed endpoint marginal $\pi \in \mathrm{P}\left(\mathcal{X}^{2}\right)$. If the BS-problem

$$
H(P \mid R) \rightarrow \min ; \quad P \in \mathrm{P}(\Omega): P_{t_{k}}=\mu_{t_{k}}, 1 \leq k \leq K, P_{01}=\pi,
$$


is such that $\inf (27)<\infty$, its unique solution $P$ is reciprocal and writes as

$$
P=\exp \left(\sum_{1 \leq k \leq K} \theta_{t_{k}}\left(X_{t_{k}}\right)+\eta\left(X_{0}, X_{1}\right)\right) R
$$

for some measurable functions $\theta_{t_{k}}: \mathcal{X} \rightarrow[-\infty, \infty), 1 \leq k \leq K$ and some measurable function $\eta: \mathcal{X}^{2} \rightarrow[-\infty, \infty)$.

Proof. See [BL]. For a more analytic proof of (26), see [Léo01].

Remark 4.8. Following Remark 2.4, it can be proved that Theorem 4.7 extends to a stochastically complete Riemannian manifold $\mathcal{X}$ with $R$ the reversible Brownian path measure having marginal $R_{0}=$ vol.

We see that (25) has the desired shape (26) with

$$
A\left(X_{\mathcal{T}}\right)=\int_{\mathcal{T}} p\left(t, X_{t}\right) \alpha(d t)
$$

and (29) holds true for the solution $P$ of the BS-problem under the hypotheses of Theorem 4.7-(b) where only finitely many time marginal constraints are considered.

Remark 4.9. In [BCN17] the authors provide, with Sinkhorn algorithm, 1 and 2-dimensional simulations for the situation of Theorem 4.7 with the difference that in their simulation, entropic minimization problem with respect to the reversible measure of Brownian motion is replaced by entropic regularisation of Euler flow. But they also prove that passing from the second to the first problem is just a question of changing the kernel between the marginal constraints: Gaussian kernel for the second one, heat kernel for the first one. So their simulations can easily be extended to the situation of Theorem 4.7. Moreover for very fine discretizations both kernels are very close one to another.

\section{Kinematics of REgular SOlutions}

In this section, the reference path measure $R$ is the law of the reversible Brownian motion with diffusion coefficient $a>0$ on $\mathcal{X}=\mathbb{T}^{n}$ or $\mathcal{X}=\mathbb{R}^{n}$, see Definition 2.1.

In view of (25) in Corollary 3.7 and (28) in Theorem 4.7, we say that (BS) admits a regular solution if it can be written as

$$
P=\exp \left(\eta\left(X_{0}, X_{1}\right)+\sum_{s \in \mathcal{S}} \theta_{s}\left(X_{s}\right)+\int_{\mathcal{T}} p\left(t, X_{t}\right) d t\right) R
$$

for some functions $\eta: \mathcal{X}^{2} \rightarrow \mathbb{R}, p: \mathcal{T} \times \mathcal{X} \rightarrow \mathbb{R}$ where $\mathcal{T} \subset[0,1]$ is a finite union of intervals and $\theta_{s}$ with $s$ running through a finite subset $\mathcal{S}=\left\{s_{k} ; 1 \leq k \leq K\right\} \subset(0,1)$ such that the function $\psi^{x}:[0,1] \times \mathcal{X} \rightarrow \mathbb{R}$ specified by (34) below is well-defined, $C^{2}$ in space and piecewise $C^{1}$ in time.

We will assume in this section that such regular solution exists. Notice that, in the important case where the BS-problem is (14), i.e. the marginal constraint is the incompressibility condition: $P_{t}=$ vol for all $t \in[0,1]$, we know by Theorem 4.7 that the solution has the form (26): $P=\exp \left(A(X)+\eta\left(X_{0}, X_{1}\right)\right) R$.

We did not succeed in proving that the additive functional writes as $A(X)=\int_{[0,1]} p\left(t, X_{t}\right) d t$ for some function $p$. Actually this problem was recently considered in $[B]$ and the pressure was shown to be defined in some space of distributions.

Remarks 5.1. 
(a) The path measure $P$ defined by (30) is the solution of a BS-problem of the form

$$
H(Q \mid R) \rightarrow \min ; \quad Q \in \mathrm{M}(\Omega):\left(Q_{t}=\mu_{t}, \forall t \in \mathcal{S} \cup \mathcal{T}\right), \quad Q_{01}=\pi
$$

(b) The expression (30) corresponds to a measure $\alpha(d t)=\mathbf{1}_{\{t \in \mathcal{T}\}} d t+\sum_{s \in \mathcal{S}} \delta_{s}(d t)$ in (25).

The kinematics of $P$. While the dynamics of $P$ is specified by formula (30) which is expressed in terms of potentials $p$ and $\theta$, its kinematic description is specified by the velocity vector field $\vec{v}^{P}$ appearing in the stochastic differential equation

$$
d X_{t}=\vec{v}_{t}^{P} d t+d M_{t}^{P}, \quad P \text {-a.s. }
$$

where $M^{P}$ is a local $P$-martingale. We are going to calculate $\vec{v}^{P}$ in terms of the potentials $\theta$ and $p$. This will permit us to establish some connection between $P$ and fluid dynamics in the special case where $R$ is the Brownian path measure with diffusion constant $a>0$.

Lemma 5.2. The velocity vector field is of the form

$$
\vec{v}_{t}^{P}=a \beta_{t}^{P}, \quad P \text {-a.s. }
$$

with $\beta^{P}$ predictable satisfying $P$-almost surely

$$
\beta_{t}^{P} \cdot d X_{t}-a / 2\left|\beta_{t}^{P}\right|^{2} d t=\mathbf{1}_{\{t \in \mathcal{S}\}} \theta_{t}+p_{t} d t+d \psi_{t}^{X_{0}}\left(X_{t}\right), \quad \forall 0 \leq t \leq 1,
$$

where we have set for any $0 \leq t \leq 1$ and $x \in \mathcal{X}$,

$$
\begin{aligned}
\psi^{x}(t, z):=\log E_{R}\left[\operatorname { e x p } \left(\eta\left(x, X_{1}\right)+\right.\right. & \sum_{s \in \mathcal{S}, s>t} \theta_{s}\left(X_{s}\right) \\
& \left.\left.+\int_{\mathcal{T} \cap(t, 1]} p\left(r, X_{r}\right) d r\right) \mid X_{t}=z\right], \quad R_{t} \text {-a.s. }
\end{aligned}
$$

Note that for $t=1$, we have $\psi^{x}(1, \cdot)=\eta(x, \cdot)$.

The identity (33) is the keystone of the computation of $\vec{v}^{P}$. It relates kinetic terms on the left-hand side with dynamical terms on the right-hand side.

Proof. Our calculation of $\vec{v}^{P}$ is done by confronting the Feynman-Kac type formula (30) with the expression

$$
\frac{d P}{d R}=\frac{d P_{0}}{d R_{0}}\left(X_{0}\right) \exp \left(\int_{[0,1]} \beta_{t}^{P} \cdot d X_{t}-\frac{a}{2} \int_{[0,1]}\left|\beta_{t}^{P}\right|^{2} d t\right), \quad P \text {-a.s. }
$$

issued from Girsanov's theory, where $\beta^{P}$ is a predictable vector field. To perform this identification, two operations are required.

(i) We disintegrate $R$ and $P$ along their initial positions, i.e. $R=\int_{\mathbb{R}^{n}} R^{x}(\cdot) R_{0}(d x)$ and $P=\int_{\mathbb{R}^{n}} P^{x}(\cdot) P_{0}(d x)$, where for any $x \in \mathbb{R}^{n}$, we denote $Q^{x}(\cdot)=Q^{x}\left(\cdot \mid X_{0}=x\right)$. The main advantage of this disintegration is that it allows to work with the Markov measures $P^{x}$, while $P$ is only reciprocal, see [BL].

(ii) The expressions (30) and (35) of $d P / d R$ are not enough. We shall need for each $0 \leq t \leq 1$, formulas for the Radon-Nikodym density

$$
\frac{d P_{[0, t]}^{x}}{d R_{[0, t]}^{x}}=E_{R^{x}}\left(\frac{d P^{x}}{d R^{x}} \mid X_{[0, t]}\right)
$$

of the restrictions to the $\sigma$-field $\sigma\left(X_{[0, t]}\right)$. 
As regards (i), denoting $b^{x}$ the drift field of the Markov measure $P^{x}$, since $d X_{t}=$ $\vec{v}_{t}^{P} d t+M_{t}^{P}, P$-a.s. and for $P_{0}$-almost all $x$ in restriction to $\left\{X_{0}=x\right\}$ we have:

$$
d X_{t}=b_{t}^{x} d t+d M_{t}^{P^{x}}=\vec{v}_{t}^{P} d t+d M_{t}^{P}=\vec{v}_{t}^{P} d t+d M_{t}^{P^{x}}, P^{x} \text {-a.s. }
$$

we see that $\vec{v}^{P}$ has the special form

$$
\vec{v}_{t}^{P}=b_{t}^{X_{0}}, \text { for a.e. } t, \quad P \text {-a.s. }
$$

This shows that it is enough to obtain the drift $b^{x}$ of the Markov measure $P^{x}$ for any $x$.

As regards (ii), Girsanov's representation (35) becomes for all $0 \leq t \leq 1$,

$$
\frac{d P_{[0, t]}^{x}}{d R_{[0, t]}^{x}}=\exp \left(\int_{0}^{t} \beta_{r}^{x} \cdot d X_{r}-\frac{a}{2} \int_{0}^{t}\left|\beta_{r}^{x}\right|^{2} d r\right), \quad P^{x} \text {-a.s. }
$$

with

$$
b_{t}^{x}=a \beta_{t}^{x}, \quad P^{x} \text {-a.s. }
$$

for some predictable process $\beta^{x}$. On the other hand, with (30), (36) and the Markov property of $R$, we obtain

$$
\frac{d P_{[0, t]}^{x}}{d R_{[0, t]}^{x}}=\exp \left(\sum_{s \in \mathcal{S}, s \leq t} \theta_{s}\left(X_{s}\right)+\int_{\mathcal{T} \cap[0, t]} p\left(r, X_{r}\right) d r+\psi_{t}^{x}\left(X_{t}\right)\right), \quad R^{x} \text {-a.s. }
$$

where $\psi$ is defined at (34). Comparing the differentials of the two expressions of $d P_{[0, t]}^{x} / d R_{[0, t]}^{x}$, we arrive at

$$
\beta_{t}^{x} \cdot d X_{t}-a / 2\left|\beta_{t}^{x}\right|^{2} d t=\mathbf{1}_{\{t \in \mathcal{S}\}} \theta_{t}+\mathbf{1}_{\{t \in \mathcal{T}\}} p_{t} d t+d \psi_{t}^{x}\left(X_{t}\right), \quad \forall 0 \leq t<1, P^{x} \text {-a.s. }
$$

which is $(33)$.

Theorem 5.3. Let us assume that the dual parameters $\eta, \theta$ and $p$ are such that $P$ is regular solution of (BS), see (30) for this notion. Then,

$$
\vec{v}_{t}^{P}\left(X_{[0, t]}\right)=\vec{v}_{t}^{P}\left(X_{0}, X_{t}\right)=a \nabla \psi_{t}^{X_{0}}\left(X_{t}\right), \quad \forall 0 \leq t \leq 1, P \text {-a.s. },
$$

where $\nabla \psi_{t}^{X_{0}}\left(X_{t}\right)$ stands for $\nabla_{z} \psi^{x}(t, z)_{\mid x=X_{0}, z=X_{t}}$ and for any $x \in \mathcal{X}, \psi^{x}$ is given by (34).

Moreover, for any $x \in \mathcal{X}, \psi^{x}$ is a classical solution of the second-order Hamilton-Jacobi equation

$$
\begin{cases}{\left[\left(\partial_{t}+a \Delta / 2\right) \psi+a|\nabla \psi|^{2} / 2+\mathbf{1}_{\{t \in \mathcal{T}\}} p\right](t, z)=0,} & 0 \leq t<1, t \notin \mathcal{S}, z \in \mathcal{X}, \\ \psi(t, \cdot)-\psi\left(t^{-}, \cdot\right)=-\theta(t, \cdot), & t \in \mathcal{S}, \\ \psi(1, \cdot)=\eta(x, \cdot), & t=1 .\end{cases}
$$

Proof. Let us work $P^{x}$-almost surely with $X_{0}=x$ fixed and by means of (37), rewrite (38) as

$$
d \psi_{t}^{x}\left(X_{t}\right)=\beta_{t}^{x} \cdot d M_{t}^{P^{x}}+\left(a\left|\beta_{t}^{x}\right|^{2} / 2-\mathbf{1}_{\{t \in \mathcal{T}\}} p_{t}\right) d t-\mathbf{1}_{\{t \in \mathcal{S}\}} \theta_{t}, \quad P^{x} \text {-a.s. }
$$

where Girsanov's theory ensures that $d M_{t}^{P^{x}}=d X_{t}-b_{t}^{x} d t$ is the increment of a local $P^{x}$-martingale. We see that $t \mapsto \psi_{t}^{x}\left(X_{t}\right)$ is a $P^{x}$-semimartingale.

Our regularity assumption ensures that $\psi^{x}$ defined at (34) verifies the following Itô formula

$$
\begin{aligned}
d \psi_{t}^{x}\left(X_{t}\right)=\left[\psi_{t}^{x}-\psi_{t^{-}}^{x}\right]\left(X_{t}\right)+\nabla \psi_{t}^{x}\left(X_{t}\right) \cdot d X_{t}+\left(\partial_{t}+a \Delta \psi_{t}^{x} / 2\right)\left(X_{t}\right) d t, & R^{x} \text {-a.s. } \\
=\left[\psi_{t}^{x}-\psi_{t^{-}}^{x}\right]\left(X_{t}\right)+\nabla \psi_{t}^{x}\left(X_{t}\right) \cdot d M_{t}^{P^{x}}+\left(\partial_{t}+a \Delta \psi_{t}^{x} / 2\right)\left(X_{t}\right) d t & \\
& +a\left|\nabla \psi_{t}^{x}\right|^{2}\left(X_{t}\right) d t, \quad P^{x} \text {-a.s. }
\end{aligned}
$$


The uniqueness of the decomposition of a semimartingale (Doob-Meyer theorem) allows for the identification of the previous two $P^{x}$-almost sure expressions of $d \psi_{t}^{x}\left(X_{t}\right)$ and gives

$$
\begin{aligned}
& \beta_{t}^{x}=\nabla \psi_{t}^{x}\left(X_{t}\right) \\
& -\mathbf{1}_{\{t \in \mathcal{S}\}} \theta_{t}\left(X_{t}\right)=\left[\psi_{t}^{x}-\psi_{t^{-}}^{x}\right]\left(X_{t}\right) \\
& a\left|\beta_{t}^{x}\right|^{2} / 2-\mathbf{1}_{\{t \in \mathcal{T}\}} p_{t}\left(X_{t}\right)=\left(\partial_{t}+a \Delta \psi_{t}^{x} / 2\right)\left(X_{t}\right)+a\left|\nabla \psi_{t}^{x}\right|^{2}\left(X_{t}\right)
\end{aligned}
$$

where the first and third equalities hold $d t d P^{x}$-almost everywhere. The second one is valid $P^{x}$-a.s. and we implicitly identified the semimartingale $\psi_{t}^{x}\left(X_{t}\right)$ with its càdlàg modification. As $\psi^{x}$ is assumed to be regular for any $x$, we obtain

$$
\begin{aligned}
\beta_{t}^{P}(\omega)=\nabla \psi_{t}^{\omega_{0}}\left(\omega_{t}\right), & t \in[0,1], \omega \in \Omega \\
{\left[\psi_{t}^{x}-\psi_{t^{-}}^{x}\right](z)=-\theta(t, z), } & t \in \mathcal{S}, x, z \in \mathcal{X}, \\
\left(\partial_{t}+a \Delta / 2\right) \psi^{x}(t, z)+a\left|\nabla \psi^{x}\right|^{2}(t, z) / 2+\mathbf{1}_{\{t \in \mathcal{T}\}} p(t, z)=0, & t \in[0,1) \backslash \mathcal{S}, x, z \in \mathcal{X} .
\end{aligned}
$$

This completes the proof of the theorem.

Fluid evolution. It is well known in classical mechanics that taking the gradient of Hamilton-Jacobi leads to the Hamiltonian equation for the momentum (Newton's equation). This is a classical integrability condition. Let us do it with the second order Hamilton-Jacobi equation (39). For the PDE part, when $t \notin \mathcal{S}$ is not an instant of shock, denoting for any $x \in \mathcal{X}$ the forward velocity of $P\left(\cdot \mid X_{0}=x\right)$ by

$$
\stackrel{\vec{v}}{\longleftrightarrow}:=\vec{v}^{P\left(\cdot \mid X_{0}=x\right)}=a \nabla \psi^{x},
$$

we obtain

$$
\begin{aligned}
0=\nabla\left[\partial_{t} \psi^{x}+a / 2\left|\nabla \psi^{x}\right|^{2}+a / 2 \Delta \psi^{x}+p\right] & =\partial_{t} \nabla \psi^{x}+a \nabla \psi^{x} \cdot \nabla\left(\nabla \psi^{x}\right)+a / 2 \Delta \nabla \psi^{x}+\nabla p \\
& =\left(\partial_{t}+\stackrel{x}{v} \cdot \nabla\right) \nabla \psi^{x}+\Delta(\stackrel{x}{v}) / 2+\nabla p
\end{aligned}
$$

and multiplying by $a$, we see that

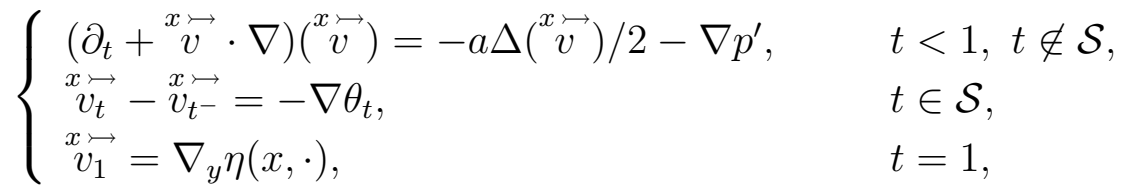

with $p^{\prime}=a p$. The left-hand side is the convective acceleration $\mathrm{D}_{t}(\stackrel{x}{\vec{v}})$ as in Navier-Stokes equation, but besides the gradient $-\nabla p^{\prime}$ of a pressure in the right-hand side, we have $-a / 2 \Delta^{x} \vec{v}$ with the wrong sign.

The forward velocity $\vec{v}$ of $P$ does not fulfill our hopes. But we are going to see that its backward velocity $\overleftarrow{v}^{P}$ does. Recall (11) and (12) for the definitions of these velocities. Let us introduce

$$
\breve{v}^{y}:=\overleftarrow{v}^{P\left(\cdot \mid X_{1}=y\right)}
$$

and for any $0 \leq \alpha \leq 1$,

$$
v_{t}^{\alpha}(z):=E_{P}\left[(1-\alpha) \stackrel{X_{0} \hookrightarrow}{v_{t}}+\alpha \stackrel{\leftarrow V_{t}}{X_{1}} \mid X_{t}=z\right]=(1-\alpha) \vec{v}_{t}(z)+\alpha \overleftarrow{v}_{t}(z)
$$

with

$$
\begin{aligned}
& \vec{v}_{t}(z):=E_{P}\left[\stackrel{X_{0} \mapsto}{v_{t}} \mid X_{t}=z\right]=\int_{\mathbb{R}^{n}} \vec{x}_{t} P\left(X_{0} \in d x \mid X_{t}=z\right) \\
& \overleftarrow{v}_{t}(z):=E_{P}\left[\stackrel{\leftrightarrow}{v_{t}} X_{1} \mid X_{t}=z\right]=\int_{\mathbb{R}^{n}} \stackrel{\leftrightarrow}{v}_{t}^{y} P\left(X_{1} \in d y \mid X_{t}=z\right)
\end{aligned}
$$


the average forward and backward velocities. In particular, $\alpha=1 / 2$ corresponds to the so-called "current velocity"

$$
v_{t}^{\mathrm{cu}}:=\left(\vec{v}_{t}+\overleftarrow{v}_{t}\right) / 2=v^{\alpha=1 / 2}
$$

The reason for calling $v^{\alpha=1 / 2}$ the current velocity is that, among all the $v^{\alpha}$ s, it is the only one satisfying the continuity equation (42) below, see (45). In contrast with the two others velocities, the current velocity transforms properly under time reversal (i.e changes its sign).

Theorem 5.4. For any $y \in \mathcal{X}$, the backward velocity field $\stackrel{\leftarrow}{v}^{y}$ of $P\left(\cdot \mid X_{1}=y\right)$ solves

$$
\begin{cases}\left(\partial_{t}+\overleftrightarrow{v}^{y} \cdot \nabla\right) \overleftrightarrow{v}^{y}=a \Delta \overleftarrow{v}^{y} / 2-\nabla p^{\prime}, & t>0, t \notin \mathcal{S}, \\ \dddot{v}_{t}^{y}-\overleftrightarrow{v}_{t^{-}}=\nabla \theta_{t}, & t \in \mathcal{S}, \\ \overleftrightarrow{v}_{0}^{y}=-\nabla_{x} \eta(\cdot, y), & t=0,\end{cases}
$$

with $p^{\prime}=$ ap and $\eta^{y}=\eta(\cdot, y)$. On the other hand, the current velocity $v^{\mathrm{cu}}$ satisfies the continuity equation

$$
\partial_{t} \mu+\nabla \cdot\left(\mu v^{\mathrm{cu}}\right)=0
$$

Moreover,

$$
\breve{v}_{t}^{y}(z)=a \nabla \varphi_{t}^{y}(z), \quad t \notin \mathcal{S},
$$

where $\varphi^{y}$ solves the Hamilton-Jacobi-Bellman equation

$$
\begin{cases}\left(\partial_{t}-a \Delta / 2\right) \varphi+a|\nabla \varphi|^{2} / 2+p=0, & t>0, t \notin \mathcal{S}, \\ \varphi(t, \cdot)-\varphi\left(t^{-}, \cdot\right)=\theta(t, \cdot), & t \in \mathcal{S}, \\ \varphi(0, \cdot)=-\eta(\cdot, y), & t=0\end{cases}
$$

The first equation of the system (41) is the desired Newton part of the Navier-Stokes equation (Burgers equation) with the right positive sign in front of the viscous force term: $a \Delta v^{y} / 2$, see (9). The continuity equation (42) is the analogue of $\nabla \cdot v=0$ in (9) which corresponds to the case $\mu \equiv 1$.

Proof. Introducing the time-reversed

$$
P^{*}:=X^{*} P
$$

of $P$, where $X_{t}^{*}:=X_{1-t}, 0 \leq t \leq 1$, we obtain $\check{v}_{t}^{P}\left(X_{[t, 1]}\right)=-\left[\vec{v}_{1-t}^{P^{*}} \circ X^{*}\right]\left(X_{[0,1-t]}\right)$. On the other hand, as $P$ is reciprocal, so is $P^{*}$. Consequently, the forward and backward velocities $\vec{v}_{t}^{P}\left(X_{[0, t]}\right)=\vec{v}^{P}\left(X_{0}, X_{t}\right)$ and $\breve{v}_{t}^{P}\left(X_{[t, 1]}\right)=-\left[\vec{v}_{1-t}^{P^{*}} \circ X^{*}\right]\left(X_{[t, 1]}\right)=-\left[\vec{v}_{1-t}^{P^{*}} \circ X^{*}\right]\left(X_{t}, X_{1}\right)=$ $\breve{v}_{t}^{P}\left(X_{t}, X_{1}\right)$ only depend on the states $X_{0}, X_{t}, X_{1}$ and can be considered simultaneously in a sum or a difference without assuming the knowledge of the whole sample path. Let us emphasize for future use the identity

$$
\breve{v}_{t}^{P}\left(X_{t}, X_{1}\right)=-\left[\vec{v}_{1-t}^{P^{*}} \circ X^{*}\right]\left(X_{t}, X_{1}\right), \quad 0 \leq t \leq 1 .
$$

Since $R$ is assumed to be reversible, i.e. $R=R^{*}$, we see that $d P^{*} / d R=\left(d P / d R^{*}\right) \circ X^{*}=$ $(d P / d R) \circ X^{*}$ and we obtain with $(30)$ that

$$
P^{*}=\exp \left(\eta^{*}\left(X_{0}, X_{1}\right)+\sum_{s \in \mathcal{S}^{*}} \theta_{s}^{*}\left(X_{s}\right)+\int_{[0,1]} p^{*}\left(t, X_{t}\right) d t\right) R
$$


with $\eta^{*}(x, y)=\eta(y, x)$ for all $x, y \in \mathcal{X}, \theta_{s}^{*}=\theta_{1-s}$ for all $s \in \mathcal{S}^{*}=\{1-s ; s \in \mathcal{S}\}$ and $p^{*}(t, \cdot)=p(1-t, \cdot)$ for all $0 \leq t \leq 1$. Applying Theorem 5.3 to $P^{*}$, we see that

$$
\vec{v}_{t}^{P^{*}}\left(X_{[0, t]}\right)=a \nabla \xi_{t}^{X_{0}}\left(X_{t}\right)
$$

with $\xi^{y}$ solution of

$$
\begin{cases}\left(\partial_{t}+a \Delta / 2\right) \xi+a|\nabla \xi|^{2} / 2+p^{*}=0, & t<1, t \notin \mathcal{S}^{*}, \\ \xi(t, \cdot)-\xi\left(t^{-}, \cdot\right)=-\theta^{*}(t, \cdot), & t \in \mathcal{S}^{*}, \\ \xi(1, \cdot)=\eta^{*}(y, \cdot), & t=1 .\end{cases}
$$

Therefore, setting $\varphi^{y}(t, \cdot)=-\xi^{y}(1-t, \cdot)$, we obtain

$$
\stackrel{\leftarrow X_{t}}{X_{t}}\left(X_{t}\right)=a \nabla \varphi_{t}^{X_{1}}\left(X_{t}\right)
$$

with $\varphi^{y}$ solution of (43). Taking the gradient of this equation and multiplying by $a$, we see that for any $y \in \mathcal{X}, \stackrel{\leftarrow}{v}^{y}$ solves (41).

Of course the marginal constraint cannot be verified by the velocities $\stackrel{x}{v}$ and $\stackrel{\leftarrow}{v}$, since they start or arrive at a Dirac mass. One must consider averages of these fields as in (40) to recover this constraint. For any smooth bounded functions, we have

$$
\begin{array}{rl}
\int_{\mathbb{R}^{n}} u & d\left(P_{t}-P_{0}\right)=E_{P} \int_{0}^{t}\left[\begin{array}{c}
X_{0} \mapsto \\
v_{s}
\end{array}\left(X_{s}\right) \cdot \nabla u\left(X_{s}\right)+\frac{1}{2} \Delta u\left(X_{s}\right)\right] d s \\
& =E_{P} \int_{0}^{t}\left[\vec{v}_{s}\left(X_{s}\right) \cdot \nabla u\left(X_{s}\right)+\frac{1}{2} \Delta u\left(X_{s}\right)\right] d s=\int_{0}^{t} d s \int_{\mathcal{X}}\left[\vec{v}_{s} \cdot \nabla u+\frac{1}{2} \Delta u\right](z) P_{s}(d z)
\end{array}
$$

and

$$
\begin{array}{rl}
\int_{\mathbb{R}^{n}} u & d\left(P_{1}-P_{t}\right)=E_{P} \int_{t}^{1}\left[\leftarrow_{v_{s}}^{X_{1}}\left(X_{s}\right) \cdot \nabla u\left(X_{s}\right)-\frac{1}{2} \Delta u\left(X_{s}\right)\right] d s \\
=E_{P} \int_{t}^{1}\left[\overleftarrow{v}_{s}\left(X_{s}\right) \cdot \nabla u\left(X_{s}\right)-\frac{1}{2} \Delta u\left(X_{s}\right)\right] d s=\int_{t}^{1} d s \int_{\mathcal{X}}\left[\overleftarrow{v}_{s} \cdot \nabla u-\frac{1}{2} \Delta u\right](z) P_{s}(d z)
\end{array}
$$

implying $\left\langle u, \partial_{t} \mu\right\rangle=\left\langle\vec{v}_{t} \cdot \nabla u+\Delta u / 2, \mu_{t}\right\rangle=\left\langle\grave{v}_{t} \cdot \nabla u-\Delta u / 2, \mu_{t}\right\rangle$. It follows that for any $0 \leq \alpha \leq 1,\left\langle u, \partial_{t} \mu_{t}\right\rangle=\left\langle v_{t}^{\alpha} \cdot \nabla u+a(1 / 2-\alpha) \Delta u, \mu_{t}\right\rangle$ which is equivalent to

$$
\partial_{t} \mu+\nabla \cdot\left(\mu v^{\alpha}\right)=a(1 / 2-\alpha) \Delta \mu \text {. }
$$

In particular, taking $\alpha=1 / 2$ leads to (42) and completes the proof of the proposition.

Remark 5.5 (The pressure does not depend on the final position $y$ ). It is an important consequence of Theorem 4.7 that the pressure $p$ and the potential $\theta$ do not depend on the final position $X_{1}$. The only explicit appearance of $X_{1}$ is in the function $\eta$. Consequently, the pressure $p^{\prime}$ in the Burgers equation (41) only depends on the actual position. This means that all the fluid particles are submitted to the same pressure field $\nabla p^{\prime}$ regardless of their final positions $y$. A similar remark is valid for the shock potential $\theta$.

Remark 5.6 (A mixture of flows tagged by their final positions). The solution $P$ of the BS-problem is well described as the statistical mixture

$$
P(\cdot)=\int_{\mathcal{X}} \stackrel{\leftarrow y}{P}(\cdot) \mu_{1}(d y)
$$

where $\stackrel{\leftarrow y}{P}:=P\left(\cdot \mid X_{1}=y\right)$ admits the gradient drift field $\stackrel{\leftarrow}{v}^{y}=\nabla \varphi^{y}$. It follows from (44) that this velocity field is completely specified by (43) where the endpoint target $y$ only occurs in the initial condition via the function $-\eta(\cdot, y)$. Formula (46) is a superposition 
principle. Each particle ending at $y$ is subject to the gradient backward velocity field

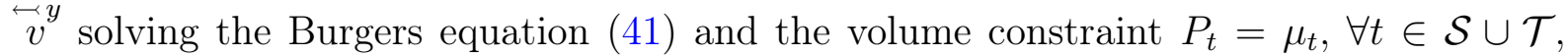
(recall (31)) is recovered superposing all the flows tagged by their final positions, via formula (46). This superposition phenomenon is very reminiscent of the structure of the multiphase vortex sheets model encountered in [Bre97].

Remark 5.7 (The average velocity is not a gradient). The incompressibility constraint applied to a gradient velocity field $v=\nabla \theta$ on the torus $\mathbb{T}^{n}$ reads as $0=\nabla \cdot v=\nabla \cdot \nabla \theta=\Delta \theta$. But this implies that $v$ vanishes everywhere. This is the reason why knowing that the average velocity is not a gradient leaves some room in our model.

We know with (44) that $\stackrel{\leftarrow}{v}_{t}^{y}(z)=\nabla_{z} \varphi^{y}(z)$ is a gradient field. Consequently, the average backward velocity writes as

$$
\overleftarrow{v}_{t}(z)=\int \nabla_{z} \varphi_{t}^{y}(z) P_{1}^{t z}(d y)
$$

and we see that the dependence on $z$ of $P_{1}^{t z}:=P\left(X_{1} \in d y \mid X_{t}=z\right)$ prevents us from identifying $\bar{v}_{t}(z)$ with $\nabla_{z}\left[\int \varphi_{t}^{y}(z) P_{1}^{t z}(d y)\right]$. Introducing the average potential

$$
\varphi_{t}^{P}(z):=\int \varphi_{t}^{y}(z) P_{1}^{t z}(d y)
$$

we obtain

$$
\overleftarrow{v}_{t}(z)=\nabla \varphi_{t}^{P}(z)-\int \varphi_{t}^{y}(z) \nabla_{z} P_{1}^{t z}(d y)
$$

\section{Existence of A SOlution on $\mathbb{T}^{n}$}

We are going to prove a sufficient condition for the existence of a solution of the BSproblem (14) in the special important case where the reference path measure $R \in \mathrm{P}(\Omega)$ is the reversible Brownian motion on the the flat torus $\mathcal{X}=\mathbb{T}^{n}$ and $\mu_{t}=$ vol, for all $t$. We refer to this problem as

$$
H(P \mid R) \rightarrow \min ;\left[P_{t}=\text { vol, } \forall 0 \leq t \leq 1\right], P_{01}=\pi .
$$

It is an adaptation of a result in [Bre89b] of existence of a generalized incompressible flow in $\mathbb{T}^{n}$. The specific property of the reversible Brownian motion $R$ is the translation invariance

$$
R=R(x+\cdot), \quad \forall x \in \mathbb{T}^{n} .
$$

Combined with the translation invariance of vol (which is implied by (47)), this will lead us to the desired result. All we have to find is some path measure $Q \in \mathrm{P}(\Omega)$ which satisfies the constraints $\left[Q_{t}=\right.$ vol, $\left.\forall 0 \leq t \leq 1\right], Q_{01}=\pi$ and such that $H(Q \mid R)<\infty$. The path measure of interest is

$$
Q=\int_{\mathcal{X}^{3}} R\left(\cdot \mid X_{0}=x, X_{1 / 2}=z, X_{1}=y\right) \gamma(d x d z d y)
$$

with $\gamma(d x d z d y)=\pi(d x d y) \operatorname{vol}(d z)$ in $\mathrm{P}\left(\mathcal{X}^{3}\right)$.

Proposition 6.1. The path measure $Q$ satisfies the constraints $\left[Q_{t}=\right.$ vol, $\left.\forall 0 \leq t \leq 1\right]$ and $Q_{01}=\pi$. If $H\left(\pi \mid R_{01}\right)<\infty$, then $H(Q \mid R)<\infty$.

Corollary 6.2. The entropy minimization problem $\left(\mathrm{H}_{\mathbb{T}^{n}}\right)$ admits a unique solution if and only if $H\left(\pi \mid R_{01}\right)<\infty$. 
Proof of Corollary 6.2. If $H\left(\pi \mid R_{01}\right)<\infty$, by Proposition 6.1 we have inf $(\mathrm{BS}) \leq H(Q \mid R)<$ $\infty$ and we conclude with Corollary 3.7-(1) that $\left(\mathrm{H}_{\mathbb{T}^{n}}\right)$ admits a unique solution. Conversely, when $\left(\mathrm{H}_{\mathbb{T}^{n}}\right)$ admits a solution $P$, we have $H\left(\pi \mid R_{01}\right)=H\left(\left(X_{0}, X_{1}\right)_{\#} P \mid\left(X_{0}, X_{1}\right)_{\#} R\right) \leq$ $H(P \mid R)<\infty$.

Proof of Proposition 6.1. As $R$ is Markov, it satisfies

$$
\begin{aligned}
& R\left(\cdot \mid X_{0}=x, X_{1 / 2}=z, X_{1}=y\right) \\
& \quad=R\left(X_{[0,1 / 2]} \in \cdot \mid X_{0}=x, X_{1 / 2}=z\right) R\left(X_{[1 / 2,1]} \in \cdot \mid X_{1 / 2}=z, X_{1}=y\right) .
\end{aligned}
$$

Let us check that $Q$ satisfies the announced constraints.

We have $Q_{01}=\pi$ since for any measurable subsets $A$ and $B$ of $\mathcal{X}$,

$$
\begin{aligned}
Q_{01}(A \times B) & =Q\left(X_{0} \in A, X_{1} \in B\right) \\
& =\int_{\mathcal{X}^{3}} R\left(X_{0} \in A \mid X_{0}=x, X_{1 / 2}=z\right) R\left(X_{1} \in B \mid X_{1 / 2}=z, X_{1}=y\right) \gamma(d x d z d y) \\
& =\int_{\mathcal{X}^{3}} \mathbf{1}_{x \in A} \mathbf{1}_{y \in B} \gamma(d x d z d y)=\gamma(A \times \mathcal{X} \times B)=\operatorname{vol}(\mathcal{X}) \pi(A \times B) \\
& =\pi(A \times B) .
\end{aligned}
$$

Let us show that for all $0 \leq t \leq 1, Q_{t}=$ vol. Take $0 \leq t \leq 1 / 2$ and denote $R\left(X_{[0,1 / 2]} \in \cdot \mid\right.$ $\left.X_{0}=x, X_{1 / 2}=z\right)=\widetilde{R}^{x, z}(\cdot)$. Since $\pi(\cdot \times \mathcal{X})=\pi(\mathcal{X} \times \cdot)=$ vol, we have $\gamma(d x d z \times \mathcal{X})=$ $\pi(d x \times \mathcal{X}) \operatorname{vol}(d z)=\operatorname{vol}(d x) \operatorname{vol}(d z)$. Hence, for any measurable bounded function $f$ on $\mathcal{X}$, we have

$$
\begin{aligned}
\int_{\mathcal{X}} f d Q_{t} & =\int_{\mathcal{X}^{3}} E_{\widetilde{R}^{x, z}}\left[f\left(X_{t}\right)\right] \gamma(d x d z d y)=\int_{\mathcal{X}^{2}} E_{\widetilde{R}^{x, z}}\left[f\left(X_{t}\right)\right] \operatorname{vol}(d x) \operatorname{vol}(d z) \\
& \stackrel{(47)}{=} \int_{\mathcal{X}^{2}} E_{\widetilde{R}^{0, z-x}}\left[f\left(X_{t}-x\right)\right] \operatorname{vol}(d x) \operatorname{vol}(d z)=\int_{\mathcal{X}^{2}} E_{\widetilde{R}^{0, a}}\left[f\left(X_{t}-x\right)\right] \operatorname{vol}(d x) \operatorname{vol}(d a) \\
& =\int_{\mathcal{X}} E_{\widetilde{R}^{0, a}}\left[\int_{\mathcal{X}} f\left(X_{t}-x\right) \operatorname{vol}(d x)\right] \operatorname{vol}(d a)=\int_{\mathcal{X}} E_{\widetilde{R}^{0, a}}\left[\int_{\mathcal{X}} f d \operatorname{vol}\right] \operatorname{vol}(d a) \\
& =\int_{\mathcal{X}} f d \operatorname{vol},
\end{aligned}
$$

where the translation invariance of vol was used at the last but one equality. This shows that $Q_{t}=$ vol for all $0 \leq t \leq 1 / 2$. A similar argument works for $1 / 2 \leq t \leq 1$.

It remains to compute the entropy $H(Q \mid R)$ to obtain a criterion of existence of a solution. Let us denote $Q_{0,1 / 2,1}(d x d z d y):=Q\left(X_{0} \in d x, X_{1 / 2} \in d z, X_{1} \in d y\right)$ and $Q^{x z y}:=$ $Q\left(\cdot \mid X_{0}=x, X_{1 / 2}=z, X_{1}=y\right)$. We have

$$
\begin{aligned}
H(Q \mid R) \stackrel{\stackrel{(i)}{=}}{H} H\left(Q_{0,1 / 2,1} \mid R_{0,1 / 2,1}\right)+\int_{\mathcal{X}^{3}} H\left(Q^{x z y} \mid R^{x z y}\right) Q_{0,1 / 2,1}(d x d z d y) \\
\stackrel{(i i)}{=} H\left(\gamma \mid R_{0,1 / 2,1}\right) \\
\stackrel{\stackrel{(i i i)}{=}}{H} H\left(\gamma_{01} \mid R_{01}\right)+\int_{\mathcal{X}^{2}} H\left(\gamma^{x y} \mid R_{1 / 2}^{x y}\right) \pi(d x d y) \\
\stackrel{(i v)}{=} H\left(\pi \mid R_{01}\right)+\int_{\mathcal{X}^{2}} H\left(\operatorname{vol} \mid R_{1 / 2}^{x y}\right) \pi(d x d y) .
\end{aligned}
$$

The factorization property of the entropy is invoked at the equalities (i) and (iii). The identity (ii) is a consequence of $Q^{x z y}=R^{x z y}$, for $\gamma=Q_{0,1 / 2,1}$-almost all $(x, z, y)$. The last equality (iv) follows from $\gamma_{01}(d x d y):=\gamma(X \in d x, Y \in d y)=\pi(d x d y) \operatorname{vol}(\mathcal{X})=\pi(d x d y)$ 
and $\gamma^{x y}(d z):=\gamma(Z \in d z \mid X=x, Y=y)=\gamma(Z \in d z)=\operatorname{vol}(d z)$ since $(X, Y)$ and $Z$ are $\gamma$-independent. It remains to show that

$$
\sup _{x, y \in \mathbb{T}^{n}} H\left(\operatorname{vol} \mid R_{1 / 2}^{x y}\right)<\infty
$$

to obtain that $H(Q \mid R)$ is finite as soon as $H\left(\pi \mid R_{01}\right)<\infty$. By means of the formula

$$
\frac{d R_{1 / 2}^{x y}}{d \mathrm{vol}}(z)=(2 / \pi)^{n / 2} \frac{\sum_{k, l \in \mathbb{Z}^{n}} \exp \left(-|z-x+k|^{2}-|y-z+l|^{2}\right)}{\sum_{k \in \mathbb{Z}^{n}} \exp \left(-|y-x+k|^{2} / 2\right)},
$$

we see that the function

$$
\begin{aligned}
H\left(\operatorname{vol} \mid R_{1 / 2}^{x y}\right)=\log \left[(\pi / 2)^{n / 2}\right. & \left.\sum_{k \in \mathbb{Z}^{n}} \exp \left(-|y-x+k|^{2} / 2\right)\right] \\
& -\int_{\mathbb{T}^{n}} \log \left(\sum_{k, l \in \mathbb{Z}^{n}} \exp \left(-|z-x+k|^{2}-|y-z+l|^{2}\right)\right) \operatorname{vol}(d z)
\end{aligned}
$$

is continuous in $(x, y)$. As $\mathbb{T}^{n}$ is compact, we have proved (48). This completes the proof of the proposition.

\section{Open PROBLEMS}

We conclude this article with a short list of related open problems.

(1) After [B], where the existence of a scalar pressure field for the BS-problem is proved, it still remains to investigate its regularity. In particular, is the gradient $\nabla p$ of the pressure an approximate differential in the sense of geometric measure theory? Note that in some context, it is shown in [Bre99] that $\nabla p$ is a locally bounded measure.

(2) By Theorem 5.4 we know that, once disintegrated with respect to the final position, the velocity field solves a collection of Burgers equations. The analogy between this decomposition, see also Remark 5.6, and the homogenized model for vortex sheets studied in [Bre97] is quite appealing. Is there any physical explanation for the appearance of the superposition of the backward velocity fields conditioned on their final positions?

(3) In the context of Euler equation, Brenier introduced in [Bre99] a velocity-valued measure on the configuration space describing the distribution in the phase space of an incompressible and non-viscous fluid, which is tightly related to the notion of measure-valued solution in the sense of DiPerna and Majda [DM87]. Would an analogous stochastic description of an incompressible viscous fluid yield interesting results?

(4) What happens in a domain with a boundary? Clearly, one must consider the law of a reflected Brownian motion as the reference law $R$. As mentioned in Remark 2.4, our necessary and sufficient condition in Proposition 2.3 which states existence and uniqueness for problem (BS) extends to Riemannian manifolds with boundary. It remains to study the kinematics of regular solutions, to extend Theorem 5.3 and 5.4 and to find the corresponding extensions of Hamilton Jacobi and Hamilton-JacobiBellman equations (39) and (43).

\section{Acknowledgements}


The second and the fourth authors were partially supported by the FCT Portuguese project PTDC/MAT-STA/0975/2014. The third author was partially granted by Labex MME-DII (ANR11-LBX-0023-01).

\section{REFERENCES}

[AAC14] A. Antoniouk, M. Arnaudon, and A.B. Cruzeiro. Generalized stochastic flows and applications to incompressible viscous fluids. Bull. Sci. Math., 138(4):565-584, 2014.

[ACF] M. Arnaudon, A.B. Cruzeiro, and S. Fang. Generalized stochastic lagrangian paths for the Navier-Stokes equation. Preprint, arXiv:1509.03491.

[AF08] L. Ambrosio and A. Figalli. On the regularity of the pressure field of Brenier's weak solutions to incompressible Euler equations. Calc. Var. Partial Differential Equations, 4:497-509, 2008.

[AF09] L. Ambrosio and A. Figalli. Geodesics in the space of measure-preserving maps and plans. Arch Rational Mech Anal, 194:421-462, 2009.

[Arn66] V. Arnold. Sur la géométrie différentielle des groupes de Lie de dimension infinie et ses applications à l'hydrodynamique des fluides parfaits. Ann. Inst. Fourier, 16(1):319-361, 1966.

[B] A. Baradat. On the existence of a scalar pressure field in the Bredinger problem. arXiv:1803.06299

[Ba19] A. Baradat. Transport optimal incompressible : dépendance aux données et régularisation entropique. PhD Thesis. Université Paris-Saclay, 2019.

[BL] A. Baradat and C. Léonard. Minimizing the relative entropy with respect to Markov and reciprocal measures. Preprint.

[BM] A. Baradat and L. Monsaingeon. Small noise limit and convexity for generalized incompressible flows, Schrödinger problems, and optimal transport. arXiv:1810.12036. To appear in Arch. Ration. Mech. Anal.

[BCN17] J.-D. Benamou, G. Carlier and L. Nenna. Generalized incompressible flows, multi-marginal transport and Sinkhorn algorithm. Numerische Mathematik, 142(1):33-54, 2017.

[Bre89a] Y. Brenier. A combinatorial algorithm for the Euler equations of incompressible flows. Computer Methods in Applied Mechanics and Engineering, 75(1-3):325-332, 1989.

[Bre89b] Y. Brenier. The least action principle and the related concept of generalized flows for incompressible perfect fluids. J. Amer. Math. Soc., 2(2):225-255, 1989.

[Bre93] Y. Brenier. The dual least action problem for an ideal, incompressible fluid. Arch. Rational Mech. Anal., 122(4):323-351, 1993.

[Bre97] Y. Brenier. A homogenized model for vortex sheets. Arch. Rational Mech. Anal., 138:319-353, 1997.

[Bre99] Y. Brenier. Minimal geodesics on groups of measure-preserving maps and generalized solutions of the Euler equations. Communications on Pure and Applied Mathematics, 52(4):411-452, 1999.

[DM87] R. J. DiPerna and A. J. Majda. Oscillations and concentrations in weak solutions of the incompressible fluid equations. Comm. Math. Phys., 108:667-689, 1987.

[EM70] D. Ebin and J. Marsden. Groups of diffeomorphisms and the motion of an incompressible fluid. Ann. Math., 92:102-163, 1970.

[FG97] H. Föllmer and N. Gantert. Entropy minimization and Schrödinger processes in infinite dimensions. Ann. Probab., 25(2):901-926, 1997.

[Fö188] H. Föllmer. Random fields and diffusion processes, in École d'été de Probabilités de Saint-Flour XV-XVII-1985-87, volume 1362 of Lecture Notes in Mathematics. Springer, Berlin, 1988.

[GL10] N. Gozlan and C. Léonard. Transport inequalities. A survey. Markov Processes and Related Fields, 16:635-736, 2010.

[Léo] C. Léonard. Lecture notes on convex optimization with some applications to probability theory. Incomplete draft at http://leonard.perso.math.cnrs.fr/.

[Léo01] C. Léonard. Minimizers of energy functionals. Acta Math. Hungar., 93(281-325), 2001.

[Léo12a] C. Léonard. From the Schrödinger problem to the Monge-Kantorovich problem. J. Funct. Anal., 262(1879-1920), 2012.

[Léo12b] C. Léonard. Girsanov theory under a finite entropy condition. In Séminaire de probabilités de Strasbourg, vol. 44., pages 429-465. Lecture Notes in Mathematics 2046. Springer, 2012.

[Léo14a] C. Léonard. Some properties of path measures. In Séminaire de probabilités de Strasbourg, vol. 46., pages 207-230. Lecture Notes in Mathematics 2123. Springer., 2014. 
[Léo14b] C. Léonard. A survey of the Schrödinger problem and some of its connections with optimal transport. Discrete Contin. Dyn. Syst. A, 34(4):1533-1574, 2014.

[Nel67] E. Nelson. Dynamical theories of Brownian motion. Princeton University Press, 1967.

[Nen16] L. Nenna. Numerical methods for multi-marginal optimal transportation. PhD Thesis. PSL Research University (Paris), 2017. https://basepub.dauphine.fr/handle/123456789/16256

[Shn85] A.I. Shnirelman. The geometry of the group of diffeomorphisms and the dynamics of an ideal incompressible fluid. Math. Sb., 128:82-109, 1985.

[Shn94] A.I. Shnirelman. Generalized fluid flows, their approximation and applications. Geom. Funct. Anal., 4(5):586-620, 1994.

[Yas83] K. Yasue. A variational principle for the Navier-Stokes equation. J. Funct. Anal., 51(2):133-141, 1983.

Institut de mathématiques de Bordeaux, Université de Bordeaux, 33405 Talence Cedex, FRANCE

E-mail address: marc.arnaudon@math.u-bordeaux.fr

GFMul and Dep. de Mat. Instituto Superior Técnico, Av. Rovisco Pais, 1049-001 LisBoA, Portugal

E-mail address: ana.cruzeiro@tecnico.ulisboa.pt

Modal'X, UPL, Univ Paris Nanterre, F92000 Nanterre France

E-mail address: christian.leonard@math.cnrs.fr

GFmul and Dep. de Mat. Faculty of Sciences. Campo Grande, Edifício C6. PT-1749016 Lisboa. Portugal

E-mail address: jczambrini@fc.ul.pt 